\title{
The near-infrared properties of the host galaxies of radio quasars
}

\author{
S. F. Sánchez ${ }^{1,2,3, \star}$ and J. I. González-Serrano ${ }^{3}$ \\ 1 Astrophysikalisches Institut Potsdam (AIP), An der Sternwarte 16, 14482 Potsdam, Germany \\ e-mail: ssanchez@aip.de \\ 2 Isaac Newton Group, Apt. 321, 38700 Santa Cruz de la Palma, Spain \\ ${ }^{3}$ Instituto de Física de Cantabria (IFCA), Universidad de Cantabria-CSIC, Avd. de Los Castros S/N, 35005-Santander, Spain \\ e-mail: gserrano@ifca.unican.es
}

Received 26 July 2002 / Accepted 4 April 2003

\begin{abstract}
We present $K$-band images of 31 radio quasars selected from the B3-VLA quasar sample. A new method has been developed to detect and restore the host galaxies of these quasars. We have detected the host galaxies of 16 of them. Using these data together with previous data from the B3-VLA quasars (Carballo et al. 1998, hereafter Paper I) and literature data from other samples, we have analyzed a sample of 69 hosts of radio quasars, covering a redshift range between $0<z<3$.

The host galaxies are large $\left(r_{\mathrm{e}} \sim 15 \mathrm{kpc}\right)$ and luminous elliptical galaxies $\left(\sim 75 \%\right.$ of them brighter than $\left.L_{K}^{*}\right)$, with an evolution similar to that of radio galaxies. A significant fraction $(\sim 40 \%)$ of them shows evidence of a possible collision/merging process. They follow a $\mu_{\mathrm{e}}-r_{\mathrm{e}}$ relation similar to that of normal elliptical galaxies. The morphological and photometric similarities between these galaxies and radio galaxies in this wide range of redshifts is a good test of the reliability of unification schemes. All of them show little evolution from $z=3$ to the present epoch. Their $K-z$ distribution is consistent with a no-evolution model, with a fraction of the dispersion due to differences in radio power. We have found a correlation between the quasar radio power and the host luminosity. We have also found a correlation between the host and nuclear source luminosities. These correlations could be induced by a physical relation between the central black hole and the bulge mass (Magorrian et al. 1998).
\end{abstract}

Key words. galaxies: quasars: general

\section{Introduction}

The studies of the host galaxies (HGs) of active galactic nuclei (AGNs) can help us to answer important questions related with these peculiar objects: (i) what is the origin of the nuclear activity? (ii) what is the origin of radio emission? (Smith \& Heckman 1990; Hutchings \& Neff 1992), (iii) which AGNs can be unified by orientation effects (standard unification schemes, Antonucci 1993; Urry \& Padovani 1995) and which not? (e.g., Ellingson et al. 1991), (iv) which is the proper model to explain the quasars evolution? (e.g., Kauffmann \& Haehnelt 2000). It is necessary to determine the space of parameters filled by the different families of AGNs in order to determine which apparently normal galaxies could contain a dormant AGN.

Studies of samples of low luminosity and low redshifts AGNs (e.g., MacKenty 1990; González-Serrano et al. 1993) showed a difference in the morphology of the host galaxies of radio loud (elliptical) and radio quiet (spiral) sources. Preliminary studies of the HGs of quasars from groundbased telescopes apparently supported this hypothesis to explain

Send offprint requests to: S. F. Sánchez,

e-mail: ssanchez@aip.de

* Visiting Astronomer, German-Spanish Astronomical Centre, Calar Alto, operated by the Max-Planck-Institute for Astronomy, Heidelberg, jointly with the Spanish National Commission for Astronomy. radio activity (e.g. Smith et al. 1986; Véron-Cetty \& Woltjer 1990). These studies are limited by the atmospheric seeing: The central point-like source contaminates the surrounding $\mathrm{HG}$, and/or is even blurred by it, due to the point spread function (PSF). Due to this, these studies are very complex, especially at high $z$, where the surface brightness of the HGs drops significantly due to cosmological dimming. Observing at the near-infrared (NIR) reduces the central point-like contribution, since the ratio between the luminosity of the nuclear source and the host is minimized (Dunlop et al. 1993). This strategy has been used by several authors (McLeod \& Rieke 1994a,b; Taylor et al. 1996; Hutchings \& Neff 1997; Kotilainen et al. 1998; Carballo et al. 1998; Aretxaga et al. 1998; Percival et al. 2001). At low $z(z \leq 0.4)$ they found that (i) the radio quasars inhabit large and luminous elliptical galaxies $\left(r_{\mathrm{e}} \sim 10 \mathrm{kpc}\right.$ and $L_{\mathrm{HGs}}>2 L^{*}$ ), and (ii) a considerable fraction of the radioquiet quasars inhabits also elliptical galaxies, and not only spiral ones, in opposition with what it was previously claimed. The most luminous quasars inhabit elliptical rather than spiral galaxies, despite their radio-loudness. Detailed morphological analysis at higher $z$ ranges was not possible. It seems that the HGs of different families of radio sources have similar morphologies (down to $z<0.4$ ) and luminosities (down to $z<1$ ), which reinforces the standard unification schemes of radio sources. 
Table 1. Characteristics of the data.

\begin{tabular}{rcccccccc}
\hline \hline $\begin{array}{r}\text { Date } \\
(1)\end{array}$ & $\begin{array}{c}\text { Telescope } \\
(2)\end{array}$ & $\begin{array}{c}\text { Instrument } \\
(3)\end{array}$ & $\begin{array}{r}\text { FOV } \\
(4)\end{array}$ & $\begin{array}{c}\text { Pixel scale } \\
(5)\end{array}$ & $\begin{array}{c}\text { Exp. time } \\
(6)\end{array}$ & $\begin{array}{c}\sigma_{K} \\
(7)\end{array}$ & $\begin{array}{c}\text { seeing } \\
(8)\end{array}$ & $\begin{array}{c}\mu_{K, \text { lim }} \\
(9)\end{array}$ \\
\hline 29 Sep. 96 & $4.2 \mathrm{~m}$ WHT & WHIRCAM & $\sim 1^{\prime} \times 1^{\prime}$ & $0.240^{\prime \prime} /$ pixel & $900 \mathrm{~s}$ & 0.03 & $0.8^{\prime \prime}$ & 21.8 \\
14 Oct. 97 & $3.5 \mathrm{~m}$ CAHA & OMEGA & $\sim 6^{\prime} \times 6^{\prime}$ & $0.396^{\prime \prime} / \mathrm{pixel}$ & $1800 \mathrm{~s}$ & 0.06 & $1.2^{\prime \prime}$ & 20.9 \\
15 Oct. 97 & $3.5 \mathrm{~m}$ CAHA & OMEGA & $\sim 6^{\prime} \times 6^{\prime}$ & $0.396^{\prime \prime} / \mathrm{pixel}$ & $1800 \mathrm{~s}$ & 0.16 & $1.1^{\prime \prime}$ & 20.9 \\
16 Oct. 97 & $3.5 \mathrm{~m}$ CAHA & OMEGA & $\sim 6^{\prime} \times 6^{\prime}$ & $0.396^{\prime \prime} / \mathrm{pixel}$ & $1800 \mathrm{~s}$ & 0.12 & $0.9^{\prime \prime}$ & 20.9 \\
3 Feb. 99 & $3.5 \mathrm{~m}$ CAHA & OMEGA & $\sim 6^{\prime} \times 6^{\prime}$ & $0.396^{\prime \prime} / \mathrm{pixel}$ & $2700 \mathrm{~s}$ & 0.09 & $1.3^{\prime \prime}$ & 21.0 \\
\hline
\end{tabular}

(1) Observing date. (2) Observing telescope. (3) NIR instrument used. (4) Field of view of the images. (5) Pixel scale. (6) Exposure time used in the science exposures. (7) Photometric error derived from the standard deviation around the mean photometric zero-point per night. (8) Mean FWHM of field stars on the images along each night. (9) Mean $3 \sigma$ limiting surface brightness magnitude of the science exposures for each night.

More detailed morphological studies have been done using the HST (e.g. Bahcall et al. 1994, 1995a,b, 1996, 1997; Hutchings et al. 1994; Disney et al. 1995; Hutchings \& Morris 1995; McLure et al. 1999; Lehnert 1999a,b; Kirhakos et al. 2001; Hutchings et al. 2002). They show that there is a large fraction of HGs with unusual and irregular morphologies, undergoing a merging process or with evidence of such a process in their recent histories. However, these results were not completely conclusive due to: (i) the saturation and undersampling of the point-like source in the HST deep images (e.g., Hutchings et al. 1995; McLure et al. 1999); (ii) strong contamination from emission lines in the optical bands at certain redshifts; (iii) the use of bands that sample the flux down to $\lambda \sim 4000 \AA$, in a range not dominated by the stellar emission, which limits the redshift range for studies that use optical bands (e.g. $z<0.6$ for the $R$-band); (iv) studies mainly based on low- $z$ samples or with a reduced number of objects. On the other hand, the few published studies using NICMOS data appear to confirm the groundbased NIR studies (e.g., McLeod \& McLeod 2001; Kukula et al. 2001; Ridgway et al. 2001).

The number of spectroscopic studies of HGs is still limited due mainly to the technical difficulties. The first attempts to determine the nature of the "Fuzz" detected around the quasars were made nearly two decades ago (Boroson \& Oke 1982, 1984, and references therein). With a large number of caveats, they found that the "Fuzz" around QSOs has an stellar nature. More recent studies (Canalizo \& Stockton 2000; Hughes et al. 2000; Nolan et al. 2001; Canalizo \& Stockton 2001; Courbin et al. 2002) have confirmed the stellar nature of the extended emission. They found that at low $z$ these objects are dominated by an old stellar population $\left(t_{\text {age }}>12 \mathrm{Gyr}\right)$, with possible traces of more recent star formation events that might be due to past interactions or even major mergers (Canalizo \& Stockton 2001; Stockton \& Ridgway 2001).

There have been few attempts to study the properties of HGs of radio loud quasars over a large and representative sample. Lehnert et al. (1999a) studied a sample of 43 3CR radio quasars, using $R$-band snapshots HST images. They detected the host for about $\sim 50 \%$ of their quasars. These HGs present distorted morphologies, close companions (in $\sim 25 \%$ of the objects), and a significant alignment with radio emission. As we quoted above, $R$-band images do not trace well the stellar population of the HGs at $z>0.6$. The use of this band combined with the depth of images (16 of the 19 undetected hosts are at $z>1$ ), and the lack of morphological classification make their results inconclusive. In contrast, McLure et al. (1999) and Dunlop et al. (2003) found, from $R$-band deep HST imaging of a sample of $z<0.4$ objects, that the morphologies of the HGs of radio-loud and radio-quiet quasars and of radio galaxies were very similar (elliptical galaxies), and that there were no significant traces of interactions and/or distorted morphologies. Their sample covers a lower range of radio powers than the one studied by Lehnert et al. (1999a). It might be impossible to generalize the properties of the HGs of powerful samples of radio quasars, like $3 \mathrm{CR}$, since these objects trace only the most violent events of the radio source family.

It is necessary to study the HGs of radio quasars in a wide range of redshifts, for complete samples with intermediate radio power, trying to trace the stellar component of their HGs. The $K$-band is the best choice to compare with other families of radio sources since they have been previous studied in this band (e.g., Lilly et al. 1985; Willot et al. 2003). We started this study in 1996 using the B3-VLA quasar sample (Vigotti et al. 1997; Sánchez et al. 2001). This sample consists of 130 radio quasars selected up to $S_{408 \mathrm{MHz}}>0.1 \mathrm{Jy}$. As part of it we published the results based on the study of the $K$-band images of 54 quasars of this sample (Carballo et al. 1998, hereafter Paper I), 32 of them were analyzed in order to detect the HG and determine its photometry. It was possible to recover the HG in 16 of them $(50 \%)$; these were large and luminous galaxies, roughly similar to radio galaxies down to $z<1$. We present here the study of new $K$-band images of 31 radio quasars from the B3-VLA sample.

The distribution of this paper is as follows: in Sect. 2 we present the sample and the observations and in Sect. 3 we show the technique used to restore the HGs of the quasars. In Sect. 4 we present the results of this analysis. In Sect. 5 we discuss the evolution of HGs of radio sources, showing its $K-z$ distribution, the structural parameters of these HGs, and its relation with radio power and nuclear emission. In Sect. 6 we study the relation between nuclear emission and radio power. We present the conclusions in Sect. 7. Throughout this article we have assumed a standard cosmology with $H_{\mathrm{o}}=50 \mathrm{~km} \mathrm{~s}^{-1} \mathrm{Mpc}^{-1}$ and $q_{\mathrm{o}}=0.5$. The selection of other cosmological parameters will not significantly affect our results ${ }^{1}$.

\footnotetext{
${ }^{1}$ A cosmology with $H_{\mathrm{o}}=65 \mathrm{~km} \mathrm{~s}^{-1} \mathrm{Mpc}^{-1}, \Omega=0.7$ and $\lambda=0.3$ will change the absolute magnitudes of the objects by $\sim 0.3$ mag in the range of redshifts we considered.
} 


\section{Observations, data reduction and photometry}

Table 1 shows a summary of the observations, including the observing nights, the telescopes and instruments used, their field of view (FOV) and pixel scale, the exposure time, the error of the photometric calibration $\left(\sigma_{K}\right.$, explained below), the mean seeing during each night (determined from the mean FWHM of the stars observed along the night) and the mean surface brightness limit at $3 \sigma$ for each night.

A standard NIR observing procedure was used: $n$ unregistered exposures of $t$ seconds each were taken and the average image registered. A number of $k$ average images was obtained following a grid pattern around the object central position, with an offset of $l$ arcseconds. Both the target and the photometric standards were observed using the same procedure. The selection of the above-mentioned parameters $(n, t, k$ and $l)$ depended on the characteristics of the detector and on the sky background level of the night. For the WHIRCAM images 75 unregistered exposures of $2 \mathrm{~s}$, and 6 average images with a 17" offset were taken, which allowed a total exposure time of $900 \mathrm{~s}$. For the October 1997 OMEGA images 25 unregistered exposures of $2 \mathrm{~s}$, and 9 average images with a $35^{\prime \prime}$ offset were taken. The procedure was repeated 4 times to reach a total exposure time of $1800 \mathrm{~s}$. For the February 1999 images the exposure time of the unregistered frames was $4 \mathrm{~s}$ and the whole procedure was repeated only three times. The total exposure time was $2700 \mathrm{~s}$.

The data were reduced using standard IRAF $^{2}$ packages. First, a dark frame, obtained before each sequence of exposures along the grid, was subtracted from each registered image. For the OMEGA images, domeflats were obtained as described in the OMEGA observer's manual. For the WHIRCAM images a skyflat was built computing the mean of all the registered images along the night, applying a $1 \sigma$ rejection algorithm to remove source contributions. These flat-field images were then used to correct the pixel-to-pixel gain variations for each registered image. The sky background frame was then obtained for each sequence of $k$ images (six for WHIRCAM, nine for OMEGA), computing the mean of these images, applying a $1 \sigma$ rejection algorithm to remove source contributions. The sky-flux frame was subtracted from each of the images of the sequence. Once sky-subtracted, the images of each sequence were re-centred and co-added. When more that one sequence was obtained for a given object, we re-centred and co-added all the sequences to obtain the final image.

All the images were obtained in photometric conditions. Flux calibration was carried out using UKIRT faint standard stars (Casali \& Hawarden 1992) observed during each night. The number of calibration exposures was always more than 12 per night for the OMEGA runs, and 4 for the WHIRCAM run (which comprised only $\sim 2.5$ hours), taken in the course of the night at different air-masses. Due to the small extinction in the NIR no air mass correction was needed, as we did not expect significant differences between the derived calibration zero-points at different air-masses. We used the mean zeropoint for each night in order to calibrate the exposures. The standard deviation around this value yields the photometric

\footnotetext{
${ }^{2}$ IRAF is distributed by the NOAO, which is operated by AURA, Inc., under contract to the NSF.
}

error due to the calibration for each night. The $K$-band magnitudes were them obtained by standard aperture photometry, selecting for each object the aperture where the flux reaches the background.

\section{Analysis of the data}

As we mention in the introduction there are various problems in the detection and restoration of the HGs of quasars at high $z$; among these we consider that the most important ones are: (i) the active nucleus dominates the emission over a wide range of wavelengths, (ii) the central source flux contaminates the HG flux, due to the width of the PSF, (iii) the angular sizes of the galaxies are smaller at $z \sim 1$ than at low $z(<0.4)$, viz. in the range of a few arcseconds, and (iv) at higher redshift, the angular size increases again, but cosmological dimming makes the surface brightness of the HGs drop significantly following a $(z+1)^{4}$-law (i.e., about 3 mag from $z \sim 0$ to $z \sim 1$ ).

We have minimalized as much as possible the effects of these problems. The first problem was reduced by the selection of the wavelength. At $\lambda \sim 1 \mu \mathrm{m}$ the SED of the galaxies presents a peak, whereas the SED of the quasars presents a minimum (Dunlop et al. 1993). Therefore, at $z \sim 1$ the ratio between $\mathrm{HG}$ and quasar fluxes is maximal in the $K$ band $\left(\lambda_{\text {eff }} \sim 2 \mu \mathrm{m}\right)$. An additional advantage is that in the NIR the HG emission is dominated by its stellar component, roughly related with the mass of the galaxy. This is in contrast with the optical bands, where the detection of extended emission around the quasars does not guarantee that the HG has been detected, since there are other more important contributions to the extended flux, such as gas flow emission (e.g., Heckman et al. 1991).

We have assumed that any extended emission detected around the quasars is associated with the stellar component of the HG. Other types of extended emission, such as radioaligned components similar to those seen in radio galaxies (Dunlop \& Peacock 1993) or synchrotron-beamed emission, might be detected in the $K$-band, although they are expected to contribute less than $10 \%$ to the HG flux (Riegler et al. 1992; Ridgway \& Stockton 1997). For the objects at $z>2$ some contribution from $\mathrm{H}_{\alpha}$ is expected, about $\sim 15 \%$ (Lehnert et al. $1999 \mathrm{~b})$. Therefore, we assume that the images of the quasars at the NIR wavelengths consist of two components only: (i) the nuclear, point-like source, characterized by a scaled PSF (to match its flux), and (ii) the host galaxy.

Our procedure to detect and recover the HG consists on the following steps:

1. We obtained for each image the surface brightness profiles (SBP) of the quasar and of a number of field stars. We masked the possible contaminating sources before obtaining the SBP. This includes basically all the sources that are more than $3^{\prime \prime}$ distant from the quasar peak and that do not show any connection such as bridges or tails with the quasar. To obtain the SBP increases the signal-to-noise ratio since it implies an average of the brightness along the eccentric anomaly. We reduce the problem to one dimension, although this implies a loss of information. This procedure 
Table 2. Sample of B3-VLA quasars studied: results of the analysis.

\begin{tabular}{|c|c|c|c|c|c|c|c|c|c|c|c|c|c|c|c|c|}
\hline $\begin{array}{l}\text { Object } \\
\text { (1) }\end{array}$ & $\begin{array}{r}z \\
(2)\end{array}$ & $\begin{array}{c}S_{408} \\
(3)\end{array}$ & $\begin{array}{r}\alpha_{408}^{1460} \\
(4)\end{array}$ & $\begin{array}{c}\text { Obs. } \\
(5)\end{array}$ & $\begin{array}{c}K \text { mag } \\
\text { (6) }\end{array}$ & $\begin{array}{r}\chi^{2} / v \\
(7)\end{array}$ & $\begin{array}{r}P_{\text {ext }} \\
(8)\end{array}$ & $\begin{array}{r}\%_{\text {ext }} \\
(9)\end{array}$ & $\begin{array}{l}\text { Ext. } \\
\text { (10) }\end{array}$ & $\begin{array}{r}\alpha \\
(11)\end{array}$ & $\begin{array}{r}P_{\mathrm{e}} \\
(12)\end{array}$ & $\begin{array}{r}P_{\mathrm{d}} \\
(13)\end{array}$ & $\begin{array}{r}P_{\mathrm{e}} / P_{\mathrm{d}} \\
\quad(14)\end{array}$ & $\begin{array}{l}K_{\mathrm{HG}} \\
(15)\end{array}$ & $\begin{array}{c}K_{\text {nuc }} \\
(16)\end{array}$ & $\begin{array}{r}r_{\mathrm{e}} \\
(17)\end{array}$ \\
\hline $0006+397$ & 1.830 & 1.15 & 0.66 & $O_{1}$ & $16.72 \pm 0.12$ & 1.98 & 95.8 & 19.5 & Yes & 0.55 & 48 & 13 & 3.7 & $18.5 \pm 0.2$ & $17.0 \pm 0.2$ & $2.39 \pm 0.09$ \\
\hline $0019+431$ & 1.050 & 2.22 & 1.34 & $O_{1}$ & $15.47 \pm 0.12$ & 0.38 & 90.4 & 5.5 & No & - & - & - & - & - & - & - \\
\hline $0022+390$ & 1.932 & 1.10 & 0.10 & $O_{1}$ & $16.65 \pm 0.13$ & 8.70 & 99.8 & 41.4 & Yes & 0.38 & 84 & 9 & 9.1 & $17.6 \pm 0.2$ & $17.2 \pm 0.1$ & $2.00 \pm 0.02$ \\
\hline $0032+423$ & 1.588 & 0.92 & 0.94 & $W$ & $16.24 \pm 0.08$ & $<0.03$ & 90.0 & $<1.0$ & No & - & - & - & - & - & - & - \\
\hline $0110+401$ & 1.479 & 1.08 & 0.65 & $O_{1}$ & $17.13 \pm 0.09$ & 4.48 & 98.6 & 51.8 & Yes & 0.40 & 84 & 11 & 7.7 & $17.9 \pm 0.2$ & $17.9 \pm 0.2$ & $3.60 \pm 0.02$ \\
\hline $0137+401$ & 1.620 & 0.82 & 0.85 & $O_{1}$ & $16.94 \pm 0.05$ & 0.91 & 91.8 & 16.5 & No & - & - & - & - & - & - & - \\
\hline $0143+446$ & 0.813 & 1.71 & 0.99 & $O_{1}$ & $13.99 \pm 0.16$ & 6.89 & 99.5 & 11.5 & $?$ & 0.40 & 84 & 11 & 7.7 & $16.3 \pm 0.1$ & $14.1 \pm 0.2$ & $7.09 \pm 0.02$ \\
\hline $0144+430$ & 1.790 & 0.92 & 1.01 & $W$ & $16.17 \pm 0.03$ & $<0.03$ & 90.0 & 15.3 & No & - & - & - & - & - & - & - \\
\hline $0144+432$ & 1.260 & 0.82 & 0.70 & $O_{1}$ & $17.12 \pm 0.18$ & 8.73 & 99.8 & 80.2 & Yes & 0.40 & 84 & 11 & 7.7 & $17.4 \pm 0.1$ & $18.9 \pm 0.4$ & $4.45 \pm 0.02$ \\
\hline $0157+442$ & 0.721 & 3.26 & 0.91 & $O_{1}$ & $16.32 \pm 0.17$ & 18.74 & 99.9 & 83.4 & Yes & 0.39 & 84 & 11 & 7.7 & $16.5 \pm 0.1$ & $18.3 \pm 0.4$ & $4.09 \pm 0.02$ \\
\hline $0158+394$ & 0.780 & 0.12 & 0.18 & $\mathrm{O}_{2}$ & $16.34 \pm 0.07$ & 5.45 & 99.1 & 6.8 & No & - & - & - & - & - & - & - \\
\hline $0217+417$ & 1.430 & 0.85 & 0.94 & $O_{1}$ & $17.18 \pm 0.15$ & 2.16 & 96.2 & $<1.0$ & No & - & - & - & - & - & - & - \\
\hline $0219+443$ & 0.852 & 0.89 & 1.17 & $W$ & $14.88 \pm 0.02$ & 2.55 & 96.7 & 42.3 & Yes & 0.51 & 61 & 13 & 4.8 & $15.8 \pm 0.1$ & $15.4 \pm 0.1$ & $1.73 \pm 0.15$ \\
\hline $0226+467$ & 1.216 & 2.45 & 0.93 & $O_{1}$ & $16.27 \pm 0.08$ & 8.23 & 99.8 & 44.7 & Yes & 0.37 & 84 & 9 & 9.1 & $17.2 \pm 0.2$ & $16.9 \pm 0.1$ & $5.25 \pm 0.02$ \\
\hline $0232+411 b$ & 0.500 & 2.75 & 0.99 & $W$ & $14.27 \pm 0.02$ & 7.63 & 99.6 & 34.2 & Yes & 0.36 & 84 & 9 & 9.1 & $15.4 \pm 0.1$ & $14.7 \pm 0.1$ & $1.44 \pm 0.01$ \\
\hline $0249+383$ & 1.120 & 0.95 & 0.34 & $W$ & $16.17 \pm 0.06$ & 4.38 & 98.5 & 46.0 & Yes & 0.60 & 40 & 17 & 2.3 & $16.8 \pm 0.1$ & $17.0 \pm 0.1$ & $2.01 \pm 0.13$ \\
\hline $0255+460$ & 1.210 & 1.74 & 0.77 & $O_{1}$ & $17.89 \pm 0.20$ & 0.30 & 90.2 & $<1.0$ & No & - & - & - & - & - & - & - \\
\hline $0704+384$ & 0.579 & 2.87 & 1.06 & $O_{1}$ & $15.02 \pm 0.16$ & 17.62 & 99.9 & 33.5 & Yes & 0.37 & 84 & 9 & 9.1 & $16.2 \pm 0.1$ & $15.5 \pm 0.2$ & $5.91 \pm 0.02$ \\
\hline $0729+391$ & 0.663 & 0.26 & 0.43 & $O_{1}$ & $15.60 \pm 0.14$ & 20.38 & 99.9 & 91.7 & Yes & 0.57 & 48 & 15 & 3.2 & $15.7 \pm 0.1$ & $18.3 \pm 0.2$ & $3.77 \pm 0.02$ \\
\hline $0740+380 \mathrm{C}$ & 1.063 & 5.55 & 1.29 & $\mathrm{O}_{2}$ & $15.62 \pm 0.05$ & 23.02 & 99.9 & 35.5 & Yes & 0.60 & 40 & 17 & 2.3 & $16.8 \pm 0.3$ & $16.1 \pm 0.2$ & $4.08 \pm 0.08$ \\
\hline $0926+388$ & 1.630 & 0.50 & 1.09 & $\mathrm{O}_{2}$ & $17.09 \pm 0.08$ & 9.95 & 99.9 & 23.6 & Yes & 0.56 & 48 & 15 & 3.2 & $18.7 \pm 0.4$ & $17.4 \pm 0.1$ & $4.05 \pm 0.14$ \\
\hline $1123+395$ & 1.470 & 0.36 & 0.82 & $\mathrm{O}_{2}$ & $15.72 \pm 0.05$ & 5.45 & 99.1 & 2.2 & No & - & - & - & - & - & - & - \\
\hline $1148+387$ & 1.303 & 1.83 & 0.94 & $\mathrm{O}_{2}$ & $14.93 \pm 0.05$ & 3.85 & 97.9 & $<1.0$ & No & - & - & - & - & - & - & - \\
\hline $1206+439 B$ & 1.400 & 5.69 & 0.85 & $\mathrm{O}_{2}$ & $15.10 \pm 0.05$ & 4.85 & 98.8 & $<1.0$ & No & - & - & - & - & - & - & - \\
\hline $1315+396$ & 1.560 & 1.16 & 0.49 & $\mathrm{O}_{2}$ & $15.93 \pm 0.06$ & 4.85 & 98.8 & 28.3 & Yes & 0.49 & 61 & 13 & 4.8 & $17.3 \pm 0.3$ & $16.3 \pm 0.1$ & $3.20 \pm 0.11$ \\
\hline $1435+383$ & 1.600 & 0.54 & 0.85 & $\mathrm{O}_{2}$ & $16.32 \pm 0.06$ & 4.25 & 98.3 & $<1.0$ & No & - & - & - & - & - & - & - \\
\hline $2311+469$ & 0.745 & 4.34 & 0.69 & $O_{1}$ & $14.79 \pm 0.06$ & 1.01 & 92.2 & 3.5 & No & - & - & - & - & - & - & - \\
\hline $2332+388$ & 3.200 & 0.42 & 0.94 & $O_{1}$ & $17.66 \pm 0.14$ & 0.68 & 91.0 & 12.5 & No & - & - & - & - & - & - & - \\
\hline $2344+429$ & 1.556 & 0.92 & 0.58 & $O_{1}$ & $16.30 \pm 0.07$ & 1.10 & 92.7 & $<1.0$ & No & - & - & - & - & - & - & - \\
\hline $2349+410$ & 2.046 & 1.38 & 0.98 & $O_{1}$ & $16.78 \pm 0.18$ & 3.18 & 97.5 & 13.8 & Yes & 0.48 & 61 & 13 & 4.8 & $19.1 \pm 0.4$ & $17.1 \pm 0.2$ & $3.35 \pm 0.02$ \\
\hline $2351+456$ & 2.000 & 2.21 & 0.27 & $O_{1}$ & $16.03 \pm 0.16$ & 6.39 & 99.4 & 17.9 & Yes & 0.39 & 84 & 11 & 7.7 & $18.0 \pm 0.2$ & $16.4 \pm 0.2$ & $2.54 \pm 0.08$ \\
\hline
\end{tabular}

(1) Object name in the B3-VLA sample. (2) Object redshift (Vigotti et al. ?). (3) Radio flux in Jy at $408 \mathrm{MHz}$. (4) Radio spectral index, $\alpha_{408}^{1460}$, defined as $S_{1460}=S_{408} \nu^{-\alpha \alpha_{408}^{140}}$. (5) Observing run: $W$, September 1996, WHT La Palma. $O_{1}$, October 1997, $3.5 \mathrm{~m}$ Calar Alto. $O_{2}$, February 1999, $3.5 \mathrm{~m}$ Calar Alto. (6) $K$-band aperture photometry. (7) $\chi^{2} / v$ of the surface brightness fitting to the PSF model. (8) percentage probability of being extended, derived from the previous $\chi^{2} / v$. (9) Contribution of the possible extension to the total flux. (10) Classification output (Yes $=$ Extended, No $=$ Not Extended). (11) Free parameter of the generalized model ( $\alpha=1$ for disk galaxies, $\alpha=0.25$ for elliptical galaxies). (12) percentage probability of being an elliptical galaxy. (13) percentage probability of being a disk galaxy. (14) Ratio of both probabilities. (15) Host $K$-band magnitude. (16) Nucleus $K$-band magnitude. (17) Effective radius of the Host in arcsec. 


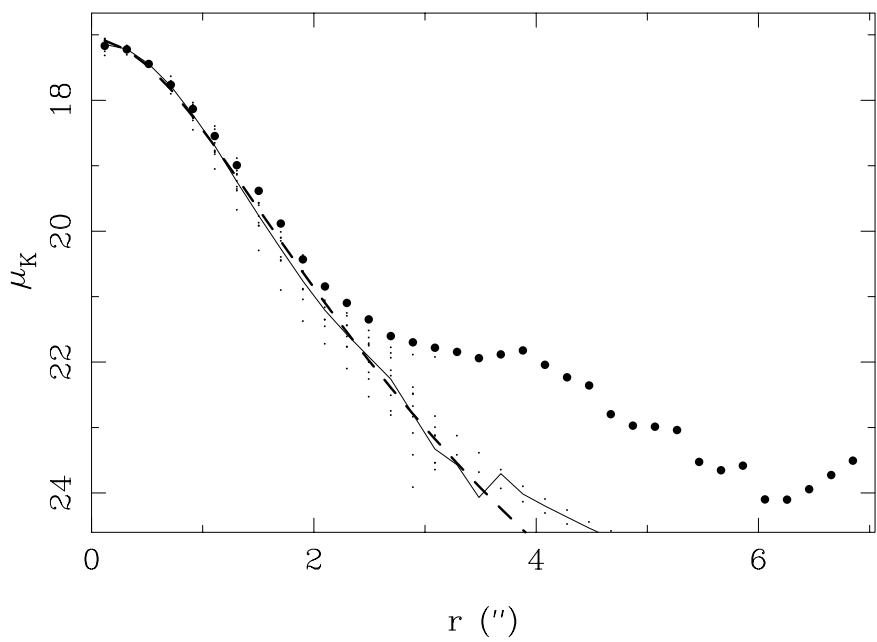

Fig. 1. Surface brightness profile (SBP) of the quasar B3 0740+380C (solid circles) together with the SBP of the field stars scaled to the quasar intensity peak (points). The solid line shows the mean of the stars' SBPs, and the dashed line shows the mean PSF derived from fitting a Moffat function.

allowed us to study the properties of HGs that were initially poorly visible in the images. The SBP was obtained using the techniques discussed in Jedrzejewski (1987), implemented in the ELLIPSE task included in the STSDAS package of IRAF.

2. The PSF was modelled by a Moffat function (Moffat 1969). The use of an analytical model allows us to give a better description of the PSF in the outer regions (normally more noisy) and let us use the PSF for a proper convolution with galaxy models (see below). To determine the best PSF model for each image we fitted the SBP of the field stars to the Moffat function

$I_{\text {Moffat }}\left(r ; I_{0}, r_{1 / 2}, \beta\right)=I_{o}\left[1+\left(2^{1 / \beta}-1\right) \cdot\left(\frac{r}{r_{1 / 2}}\right)^{2}\right]^{-\beta}$.

The width of the central core is determined by $r_{1 / 2}$, and $\beta$ determines the extension of the wings. $I_{0}$ is the scaling factor that determines the peak of the intensity. Once the field stars are fitted to this model we obtain a mean value for both $r_{1 / 2}$ and $\beta$. These values characterize the mean PSF in the image.

It was found that about $\sim 10$ stars per field were enough to determine the PSF parameters. There were enough stars per field in the OMEGA images, due to their large field of view. However, the WHIRCAM images have only 1 or 2 stars per field; still, these were found to present a stable PSF image-to-image, mainly due to the short time period in which they were obtained ( $<2.5$ hours). Therefore, we used all the stars available in all the WHIRCAM images to determine the PSF parameters.

3. The mean PSF was used to determine the probability that a quasar was extended by fitting the surface brightness profile of the quasar to the function: $\operatorname{PS} F\left(r ; I_{0}\right)=$ $I_{\text {Moffat }}\left(r ; I_{0}, r_{1 / 2}, \hat{\beta}\right)$. This method was tested on 200 simulated stars, with the same characteristics of seeing, brightness and signal-to-noise as the observed quasars. This yields a probability distribution that the surface brightness of a star fits the mean PSF, a distribution that can be used to estimate the probability that a quasar is extended or not. We have applied the same test to the field stars, finding that they fit the mean PSF model within a $95 \%$ probability, as expected. We also found that when an artificial extension is detected in a field star, it always contributes less than $10 \%$ to the total star flux.

Figure 1 ilustrates this procedure. We show the SBP of the quasar B3 $0740+380 \mathrm{C}$, together with the scaled SBP of the field stars used to determine the mean PSF. There is a dispersion of the individual star SBPs around the mean PSF. The quasar profile is further from the mean PSF than the field stars, and shows a clear extension. This difference in the SBP compared with the differences found for the stars can be used to set a probability for the extension to be real.

4. The surface brightness profile of the quasars was then fitted to a two-component model: a free-scale mean PSF model plus a galactic model. We have initially restricted ourselved to the two most commonly used analytical prescriptions for the galactic model: an exponential Freeman (1970) law describing disk galaxies and a de Vaucouleurs (1948) $r^{1 / 4}$ law describing elliptical galaxies. The fitting procedure has three free parameters: the nuclear component flux, the host galaxy flux, and the galaxy scale (for each analytical model). The galactic profile was convolved during each step of the fitting procedure by the mean PSF (normalized to one), in order to take into account the effects of the seeing on the shape of the profiles. In general, the nuclear component has a more intense surface brightness, with larger signal-to-noise ratio. Therefore, we assume that with this method we are able to recover the flux of the nuclear component better than that of the extended component. We then estimate the HG flux by subtracting the recovered nuclear component flux from the total flux.

5. We found that the quasar profile fits both models well. In order to investigate this we applied the procedure to a sample of 1000 simulated quasars. These simulated quasars consist on a central point-like source, following a Moffat function, plus an extended source, following an exponential or a $r^{1 / 4}$ function. These images cover different ranges of the parameter space, such as different ratios between the central and the extended emission, between the effective radius and the seeing, and the signal to noise of the total source. They reproduce the basic parameters of the real data, such as background noise, pixel scale, and image depth. The simulations are explained in Sánchez (2001) and they will be published in a separate paper (Sánchez 2003).

They show that it is not possible in our range of parameters to distinguish between a spiral or an elliptical HG by the comparison of the $\chi^{2} / v$ resulting from the profile fitting to the two above-mentioned models. This problem has been described before (McLure et al. 1999). This could be due to the larger signal-to-noise of the central region, which dominates the fits to such an extent that subtle differences in the extended emission become indistinguishable. We also found that the percentages of flux recovered for both the 
HG and the nuclear source are quite similar, independent of the model.

6. To solve this problem we followed a different approach: (i) the HG surface brightness profile was obtained subtracting the mean PSF model, scaled to the nuclear source flux (previously obtained), and (ii) this profile was fitted to a generalized galaxy model $\left(I_{\text {gal }} \propto \mathrm{e}^{r^{\alpha}}\right)$. This model includes the other two as particular cases: the exponential model is recovered if $\alpha=1$, and the $r^{1 / 4}$-model if $\alpha=1 / 4$. The values of $\alpha$ obtained with this method were used to classify the HGs. This method was applied to the simulated images, and yielded sharply different distributions of the $\alpha$ parameter for the spiral and for the elliptical galaxies (Fig. 2). We have used the obtained $\alpha$ to classify the HG morphologically. A similar procedure has been applied by McLure et al. (1999) to distingue between exponential and $r^{1 / 4}$ models.

The idea behind this method is that the most important region to distinguish between an elliptical and a disk galaxy is the galaxy core. The core is severely affected by the nuclear component substraction. Even in the case of a perfect subtraction there is an increase of the noise: The photon noise in this area is the sum of the photon noise from the galaxy and the QSO nucleus. Therefore, there is a loss of information in the inner part. Indeed, it is noticed in the simulations that the preferred $\alpha$ parameters of a simulated elliptical or spiral galaxy were not the expected values $(0.25$ and 1.0 , respectively) but somewhat larger ones ( 0.3 and 1.15 , respectively). Effects like this have been noticed by other authors (Meurer et al. 1995; Whitmore et al. 1999). If we force the model to fit the data to the theoretical values, both of them will fit equally badly, and cannot distinguish between the models. However, letting $\alpha$ free to be fitted we can recover the parameter that fits the data best.

In Paper I we used a rather different method to detect and restore the HGs. Before the surface brightness profile of the quasars was obtained, the images were deconvolved by a mean PSF. The brightness profiles were then obtained and fitted to different models: a Gaussian function (which characterizes the deconvolved point-like sources) and a Gaussian. plus. galactic model (which characterizes a point-like plus an extended source). This method had to be applied due to the lower quality of the data used in Paper I. In order to compare the results obtanied in Paper I with the present results, and to set limits to both methods, we have applied both of them to the simulations mentioned above.

We found that both methods are able to recover the flux of the HGs, although in different ranges of parameters. The method used in this article is valid for HGs for which the contribution to the total flux was larger than $\sim 9 \%(\sim 12 \%$ for the method used in Paper I). In this range of fluxes, the magnitudes of the HGs are recovered with an error of about $\sim 0.26$ mag ( $\sim 0.52 \mathrm{mag}$ for the method used in Paper I). There is no tendency to over- or underestimate the flux down to $K_{\mathrm{HG}} \sim 18.5$ mag ( $\sim 17$ for Paper I). Moreover, with the method presented here it is possible to recover the effective radius of the $\mathrm{HG}$, with an error of $\sim 25 \%$ (a parameter that we could not recover in Paper I). However, the current method requires

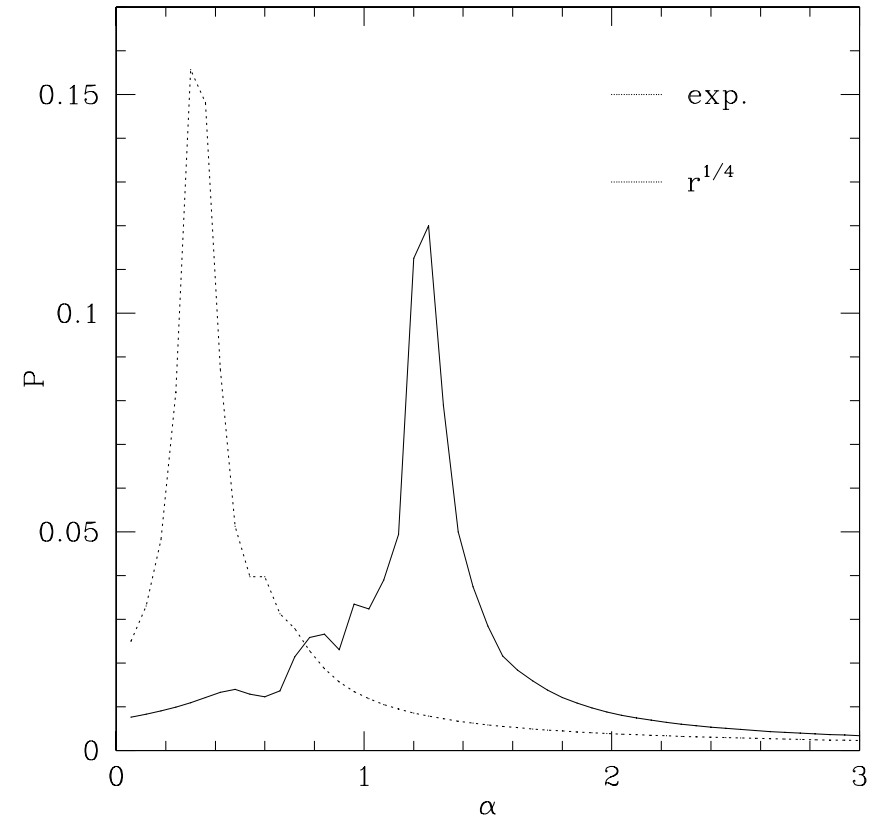

Fig. 2. Probability density functions for exponent $\alpha$ obtained from fits to simulated elliptical and disk galaxies.

(i) a good sampling of the PSF, using 5-7 stars with the same brightness as the quasars, or a more reduced number of brighter stars, and (ii) that the sizes of the HGs are similar to or larger than the seeing, which, for quasars at $z \sim 1$ limits the use of this method to good-seeing images (i.e., better than $\sim 1.3^{\prime \prime}$ ). The method used in Paper I, based on a deconvolution process, is still valid for lesser-quality images. It does not require a precise sampling of PSF and it can be used for images with seeing larger than 1.3", although it recovers the flux of the HG with larger uncertainties (0.52 mag) and for brighter HGs (>12\%).

\section{Results of the analysis}

Table 2 lists the sample of objects, including some basic parameters, such as the redshift and radio properties, and the $K$-band photometry. We have also listed the results from the analysis. A QSO has been classified as extended whenever (1) the probability that its SBP deviates from the mean PSF profile is larger than $95 \%$ and (2) the contribution of the extended source to the total flux is larger than $9 \%$. These criteria have been selected based on the results from the simulations and our analysis over the field stars.

We found an extension in 16 of the 31 quasars (55\% of the sample). The extension around B3 $0143+446$ is considered dubious, although it satisfies the criteria. This object shows a nearby star at less than 6" which could affect its SBP even when a proper mask has been applied. We will not include this object in our further analysis. Ten QSOs have a probability of being extended of more than $99 \%$. The remaining 6 quasars have probabilities between $95 \%$ and $99 \%$. Of theses 6 , four are in the range of the faintest detected HGs ( $\left.K_{\mathrm{HG}}>18 \mathrm{mag}\right)$. The fraction of detected HGs decreases with redshift, being $75 \%$ of the sample at $z<1$ and $48 \%$ at $z>1$. Beyond this redshift the fraction of detected galaxies is roughly constant. This result is 
expected since at high redshift both the surface brightness and apparent luminosity of the HGs decrease, which makes their detection difficult. We have not set a magnitude limit for the undetected host galaxies, since the detection does not depend only on the brightness of the source.

All the HGs have a larger probability of being elliptical galaxies than of being spirals. For an individual HG the probability that it is a spiral is $7-17 \%$ and that it is and elliptical is $40-84 \%$. This is the first time that a morphological analysis of the HGs of a sample of radio quasars yields a conclusive result beyond $z>0.4$. Previous results (Kotilainen \& Falomo 2000; Kotilainen et al. 1998; Kukula et al. 2001) directly assume a certain profile $\left(r^{1 / 4}\right)$, or have inconclusive results from their analysis. Our result shows that the HGs of radio quasars at high redshift have the same morphology as the radio galaxies, supporting the unification schemes of both radio sources. Similar results were found by Taylor et al. (1996), McLure et al. (1999) and Dunlop et al. (2003), at low redshift $(z<0.4)$.

The photometry of seven of these objects was presented in Paper I. The differences between the $K$-band magnitudes are within the errors $(\sim 0.3 \mathrm{mag})$. In four of them it was possible to follow a morphological analysis. Three have an extension detected in both studies (B3 0704+384, B3 0740+380C and B3 1315+396). The differences between their HG magnitudes are $-0.5,-0.4$ and 0.7 (respectively), with a mean difference of $0.05 \pm 0.65 \mathrm{mag}$. These values are within the expected errors ( $\sim 0.5 \mathrm{mag}$ for Paper I HG magnitudes, and $\sim 0.2 \mathrm{mag}$ for current data). The extension of the remaining quasar (B3 $0926+388$ ) has been detected in the present study only, since its magnitude is much fainter than the detection limit for HGs reported in Paper I.

The HG contribution to the total flux ranges between $14 \%$ (B3 2349+410) and 92\% (B3 0729+391), with a mean contribution of $\sim 41 \%$. Similar values have been reported in Paper I (range between 18\%-83\% and mean $\sim 50 \%$ ), and studies based on lower redshift samples for similar wavelengths (Taylor et al. 1996; Kotilainen et al. 1998). These values are lower at optical wavelengths, $\leq 20 \%$ for $R$-band images (e.g., Lehnert et al. 1999a; McLure et al. 1999). Therefore, the extended emission is redder than the nuclear emission. This result is expected if a stellar component dominates the extended emission rather than other contributions (such as scattered flux from the nucleus). Recent results based on off-nuclear spectroscopy have confirmed its stellar nature (Canalizo \& Stockton 2000; Nolan et al. 2001; Canalizo \& Stockton 2001; Courbin et al. 2002).

We have obtained a restored image of the HG by subtracting an image of the nuclear point-like source. This image was built assuming a Moffat function with the mean-PSF parameters (obtained by fitting to the field stars). Its flux was fixed to the value obtained from the fitting procedure for the nuclear component. The image was built using the package ARTDATA implemented in IRAF. Figure 3 shows the contour plots and surface brightness profiles for the sources with detected extension. In each panel (a) shows the contour plot (left) and profile (right) of the original source, and (b) shows the contour plot (left) and profile (right) of the recovered host galaxy. The solid line in (a) shows the surface brighteness profile of the PSF scaled to the quasar peak. The $\chi^{2} / v$ from the comparison of both profiles and the probability of being extended have also been included for each quasar. It is clearly seen that the objects present a significant extension from the comparison of both profiles. The orientation of the contour plots is North (up) and East (left), and the field-of-view corresponds to $16^{\prime \prime} \times 16^{\prime \prime}$. We have plotted the raw data before masking for nearby companions. In some cases (e.g., B3 0006+397, B3 0110+401) there are close companions that affect the unmasked SBP. However, the extension is clearly seen in spatial regions not affected by these companions. This is not the case for quasar B3 $0143+466$, classified as dubious.

Seven of the 16 objects present distortions, tails or bridges with possible close companions at $<30^{\prime \prime}$ ( $40 \%$ of the sample). These distortions could be traces of past collisions, interactions or even merging processes, which affect the host and nearby galaxies. In some of them, B3 0219+443 and B3 0740+380C, the optical-to-NIR colours of the nearby objects $\left(<30^{\prime \prime}\right)$ are compatible with the colours of galaxies at the redshift of the quasars (Sánchez 2001; Sánchez \& González-Serrano 2002). However, a contamination from a nearby object, either a galaxy or a faint star, within $\sim 2$ " of the quasar could produce a similar effect and create an artificial distorted HG. We have estimated the mean density of objects in our images using the number of objects detected in the February 1999 images (the deepest images with the larger field of view). We found a density of $2.7 \pm 0.6 \times 10^{-3} \mathrm{obj} / \operatorname{arcsec}^{2}$, including both stars and galaxies. The large dispersion is due to the fact that these objects tend to inhabit clusters or groups of galaxies of different populations (see Sánchez \& González-Serrano 2002, also for a detailed explanation of the procedure to determine the density). Using these numbers, we expect to find 0.043 objects within a box of $4^{\prime \prime} \times 4^{\prime \prime}$ around each quasars. I.e., we expect that a contaminating source could create an artificial distorted $\mathrm{HG}$ in $4.3 \%$ of the objects. For our sample of 16 extended sources we do not expect any significant contribution from this effect ( $\sim 0.64$ possible contaminated objects).

Together with data from Paper I, we have a collection of 29 HGs obtained from the analysis of the $K$-band images of 60 radio quasars from the B3-VLA sample. Fifteen of them (Paper I) show evidence of possible interactions ( $\sim 50 \%$ of the sample). This evidence is tenuous and should be confirmed by spectroscopic studies of both the host galaxies and the nearby companions.

\section{The properties of the HGs of radio sources}

\subsection{The $K-z$ distribution: The evolution of the HGs}

Figure 4 shows the $K$-band apparent magnitude of HGs of radio quasars as a function of redshift. The solid circles represent the HGs presented in this study, and the open circles the HGs presented in Paper I. The remaining points represent data obtained from the literature: diamonds from Lehnert et al. (1992), open squares from Taylor et al. (1996), asterisks from Kotilainen et al. (1998), stars from Kotilainen \& Falomo (2000), triangles from Falomo et al. (2001) and solid squares from Kukula et al. (2001). The HG magnitudes from Lehnert et al. (1992) were corrected by 0.4 mag to take into account the 
(a)

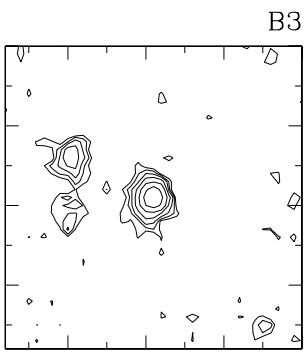

(b)

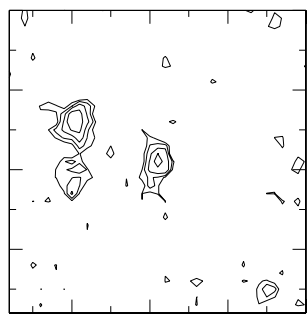

$30006+397$
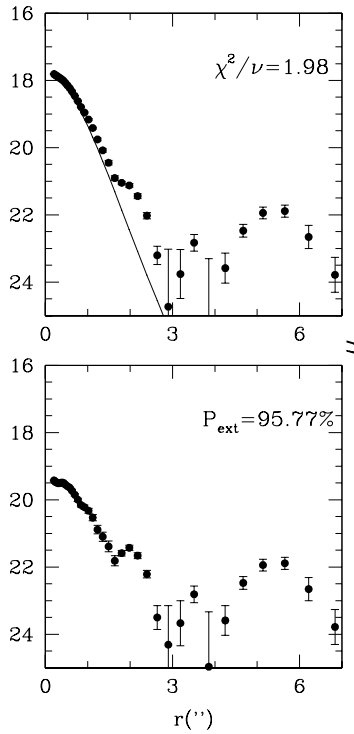

B3 $0110+401$
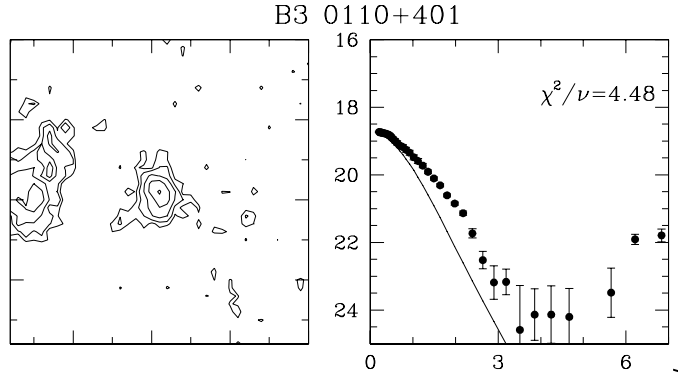

(b)
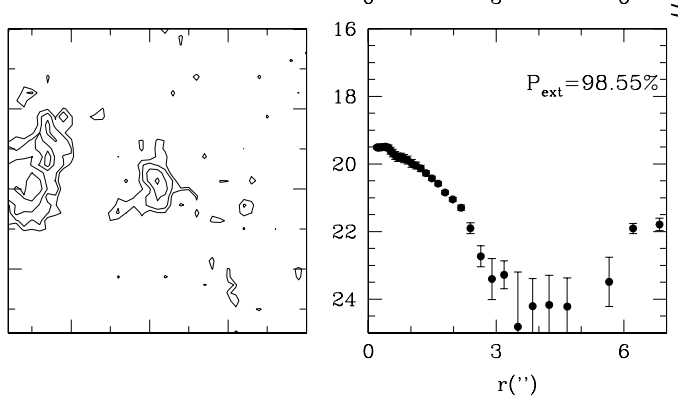

(a)

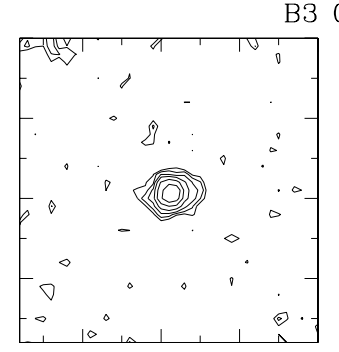

$30022+390$

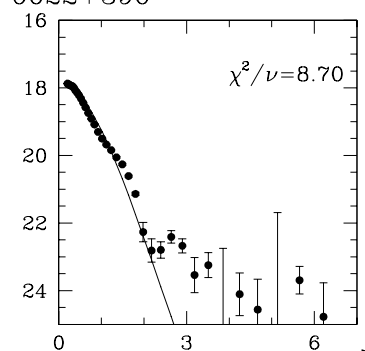

(b)

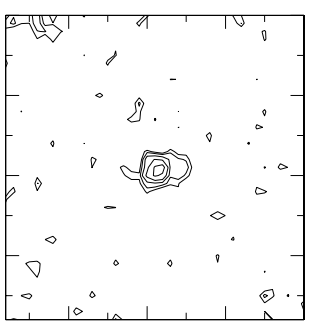

B3 $0144+432$

(a)
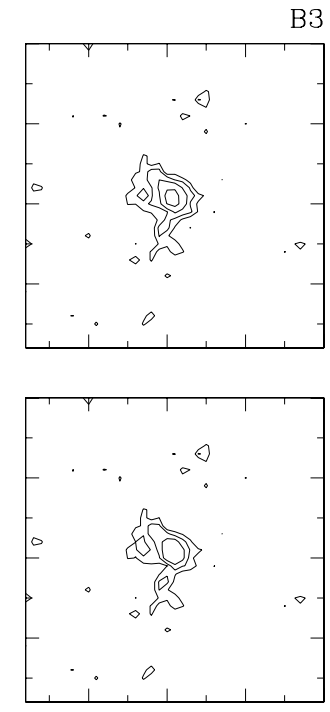
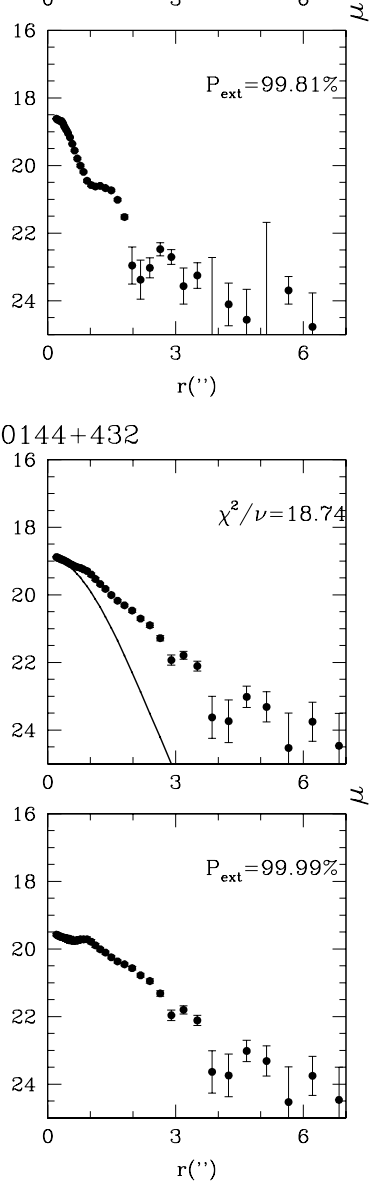

Fig. 3. Contour maps and surface brightness profiles of the quasars with detected extension. In each panel a) is the contour map (left) and surface brightness (right) of the original image, and b) is the contour map (left) and surface brigthness (right) of the HG image. The solid line in a) shows the mean PSF scaled to the quasar intensity peak. The distances between small marks are $2^{\prime \prime}$ (for objects observed with OMEGA), and $1.2^{\prime \prime}$ (for objects observed with WHIRCAM). The size of the contour maps is $16^{\prime \prime} \times 16^{\prime \prime}$ for all the images. First contour level is at $2 \sigma$ per pixel, with a separation of 0.5 mag between successive contours.

underestimation of the HG flux due to the total subtraction of the central point-like source, following the indications of the authors. Magnitudes from Kotilainen et al. (1998), Kotilainen \& Falomo (2000), Falomo et al. (2001) and Kukula et al. (2001) were transformed from $H$ to $K$ band using the correction suggested by authors $(H-K \sim 0.2 \mathrm{mag}$, Kotilainen et al. 1998). We have used only the 2 HGs detected in the $H$-band from Kukula et al. (2001) with a reliable result in the radial profile fitting. We have not considered their detections in the $J$-band (due to the uncertainties in the transformation to the $K$-band), and their dubious detections. This comprises a heterogeneous sample of 69 HGs, 29 B3-VLA HGs plus 40 HGs extracted from the literature.
The shaded region shows the space occupied by the radio galaxies of different samples $( \pm 1.5 \sigma$ from the mean value), including the 3C and B2 (Class 1Jy) samples, studied by Lilly et al. (1985) and Lilly (1989), the 6C and 7C sample, and the HzRG (High- $z$ radio galaxies). The distribution of the mean value is represented by the dashed line. All the radio galaxy data have been extracted from Fig. 1 of van Breugel et al. (1999), except for the 7C data, obtained from Lacy et al. (2000) and Willot (2003). These radio galaxy samples, except for $\mathrm{HzRG}$, are roughly complete (i.e. $3 \mathrm{C}$ is $98 \%$ complete). In contrast, only low- $z$ quasar host samples are complete (e.g. Taylor et al. 1996). The quasar hosts are mainly $\sim 50 \%$ complete, as only the brightest objects are detected. 
(a)

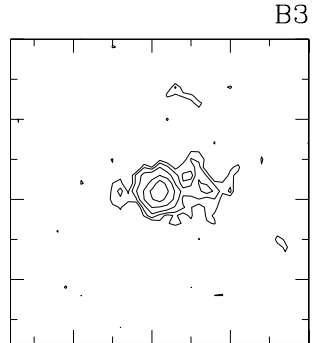

$0157+442$

(b)

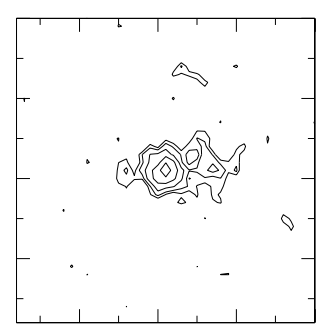

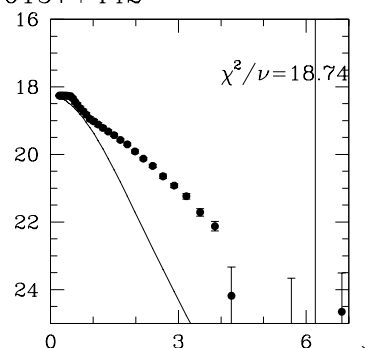
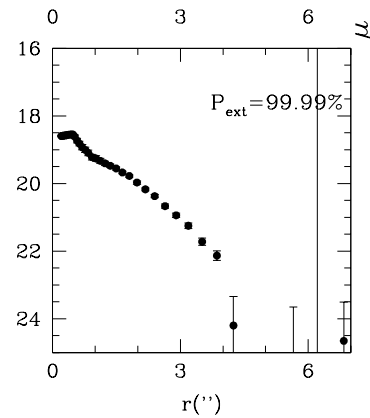

B3 $0226+467$

(a)
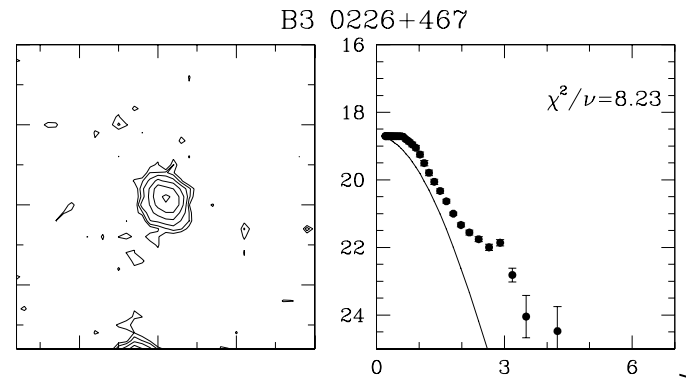

(b)
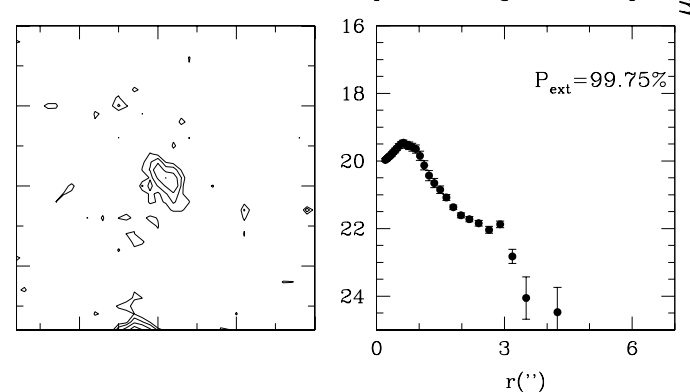

(a)

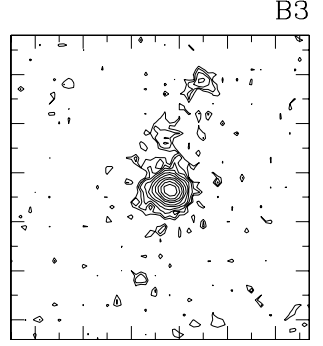

B3 $0219+443$

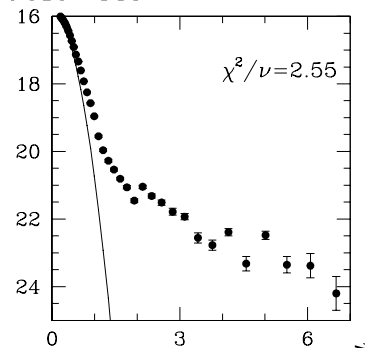

(b)
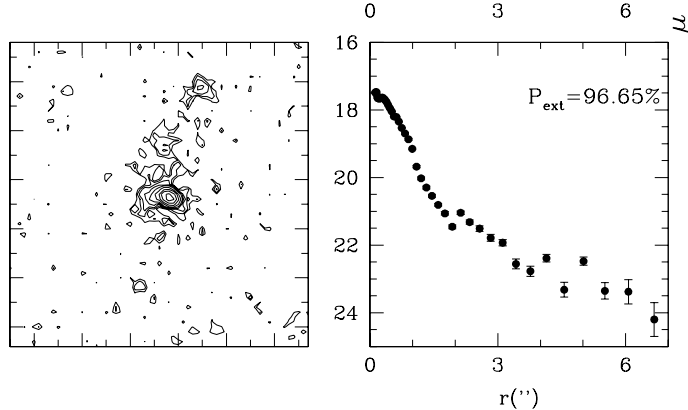

B3 $0232+411 b$

(a)
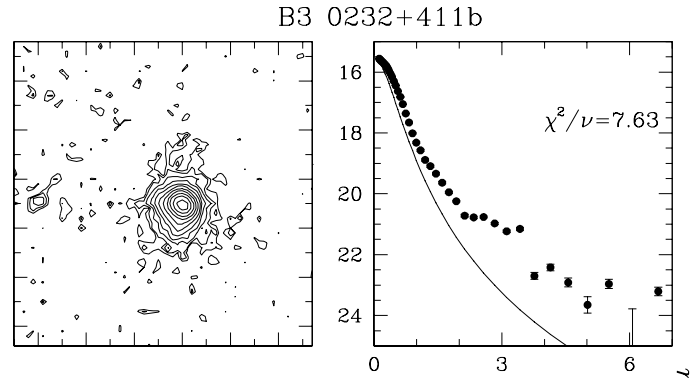

(b)
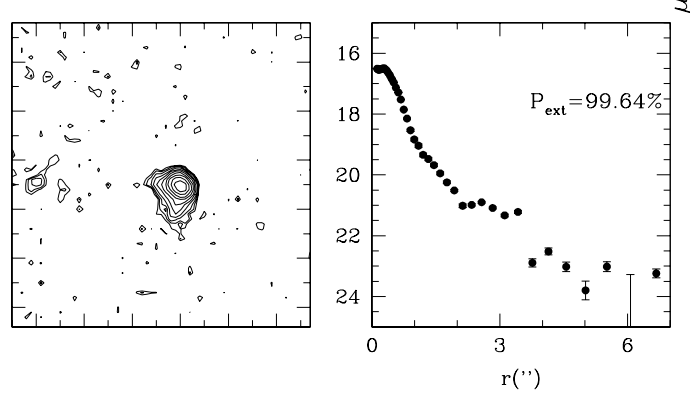

Fig. 3. continued.

The distributions are roughly similar. Therefore, the HGs of different families of radio sources have similar luminosities at different cosmological epochs, down to $z<3$. However, 9 of the $70 \mathrm{HGs}$ presented in this figure are clearly more luminous than the radio galaxies ( $\sim 13 \%$ of the HGs sample), 6 of them at $z>1$. Considering also that only half of the HGs of the radio quasars have been recovered (or even less at $z>1$, as we quoted above), we conclude that although the mean distributions seem to agree, the dispersion along the mean value is larger for the HGs. The errors of the HG magnitudes (upper-left box) are clearly larger than the errors of the magnitudes of the radio galaxies. This could account for the larger dispersion in the distribution in Fig. 4. But not all the difference could be due to this cause: the largest errors were about $0.5 \mathrm{mag}$, and the difference between the magnitudes of the high-luminosity HGs and brightest radio galaxies at the same redshift was $\sim 1-1.5$ mag. We need to explore other possibilities to explain these extreme luminosities.

Different evolutionary models are shown in Fig. 4. Each solid concave line shows the evolution of an elliptical galaxy with different formation redshifts $\left(z_{\text {for }}=7,4.5,2.5,1.5,1.0\right.$ and 0.7 , from right to left). Each convex line shows the evolution of a galaxy in a continuous burst process with different formation redshifts $\left(z_{\text {for }}=2.5,1.5,1.0\right.$ and 0.7 , from right to left). These models have been included to show the most extreme situations, from pasive to violent evolution, and from old to young galaxies. The solid near-straight line shows the magnitudes for a galaxy without evolution, which could be considered the most extreme situation of a very old galaxy without present star formation. The evolution tracks have been determined using the GISSEL code (Bruzual \& Charlot 1993), assuming an intrinsic luminosity of $M_{*}$ (Mobasher et al. 1993), and a Salpeter mass distribution with a range of masses between 0.1 and $125 M_{\odot}$ (Salpeter 1955). The intrinsic luminosity has been selected to match the data at low $z$ for both families of HGs. We have used 
(a)

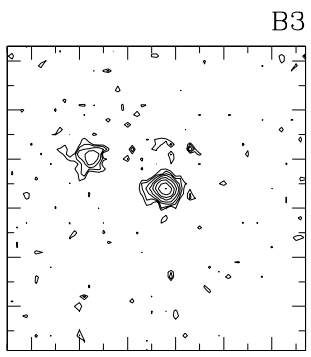

(b)

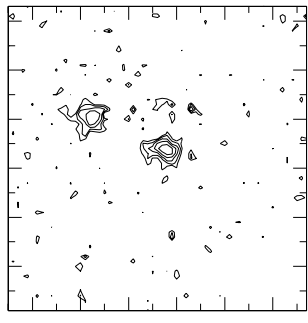

$0249+383$
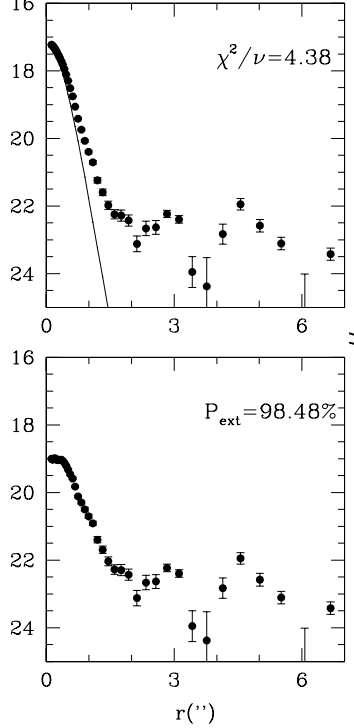

$r(")$

(a)
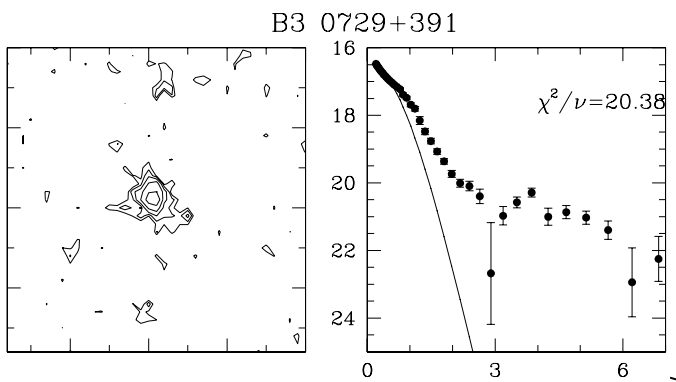

(b)
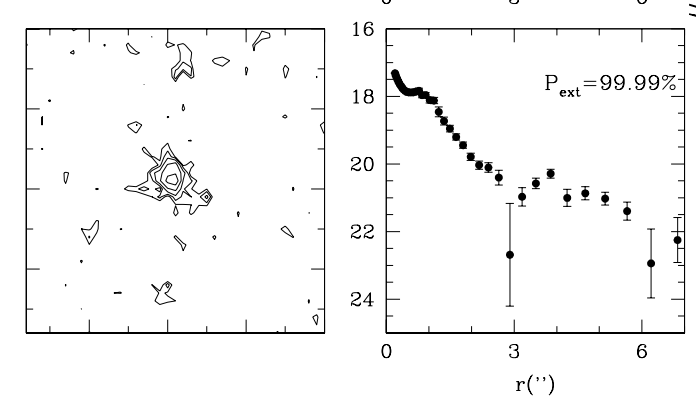

Fig. 3. continued.

the E-type and Burst-type isochrone spectral energy distributions (ISED) from Bolzonella et al. (2000).

The mean distribution of magnitudes with redshift is well described by an old E-type galaxy model, near to a noevolution model. However, there is a large dispersion around this mean track. The dispersion could be explained by a range of formation redshifts, a range of different evolutions and/or a range of intrinsic luminosities (see figure). In the first two cases, as we explained in Paper I, the brightest HGs would be galaxies in a violent star formation process. These galaxies would be blue and their predicted optical magnitudes would be as bright as (or even brighter than) the observed quasar magnitudes. Therefore, a large range of star-formation redshifts or violent evolution have to be ruled out as a general explanation of the data. In the same way, Nolan et al. (2001) and de Vries et al. (2000) have presented evidence that low- $z$ quasar hosts and radio galaxies are dominated by a stellar population of at

(a)

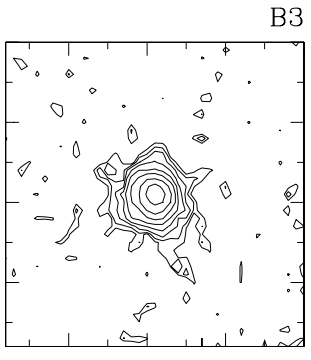

$30704+384$

(b)
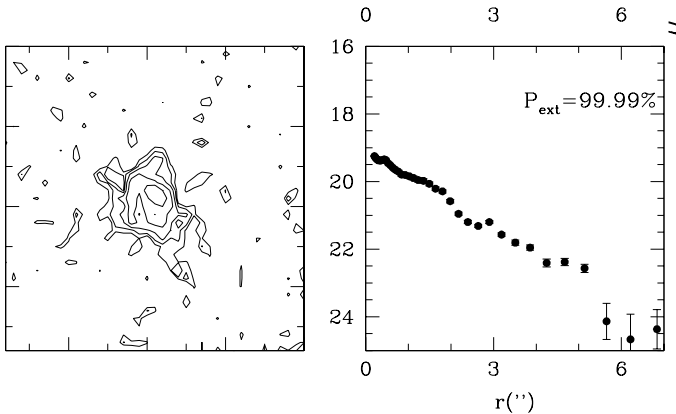

B3 $0740+380 \mathrm{C}$

(a)
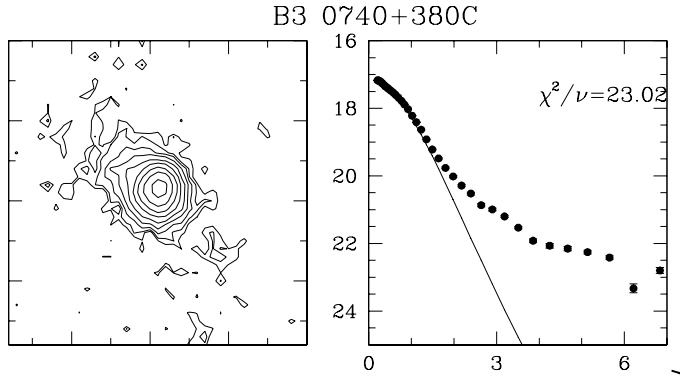

(b)
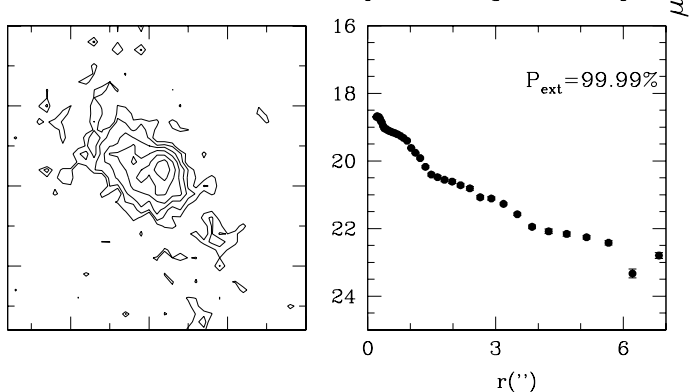

least $\sim 12$ Gyr. It appears more likely that the dispersion in the distribution is due to a dispersion in the instrinsic luminosity of the HGs. This dispersion seems to be larger for the HGs of radio quasars than for radio galaxies.

Eales \& Rawlings (1996), Rawlings (1998), and more recently Willot et al. (2003) found that the low radio-luminosity radio galaxies $(6 \mathrm{C}$ and $7 \mathrm{C}$ ) are fainter at the NIR than powerful ones (3C and B2) at the same redshift. This implies a correlation between the radio power and the NIR luminosity of the galaxy. Its effect on the $K-z$ distribution is an increase in the dispersion. Therefore, the $K-z$ distribution for the different families of radio sources is well described by a mean evolution typical for an old E-type galaxy (or a no-evolution model), and a range of absolute luminosities correlated with the radio power. The reason for this correlation could be or relation of both parameters (radio power and HG luminosity) with the central black hole mass (McLure et al. 1999; Dunlop et al. 2003). 
(a)
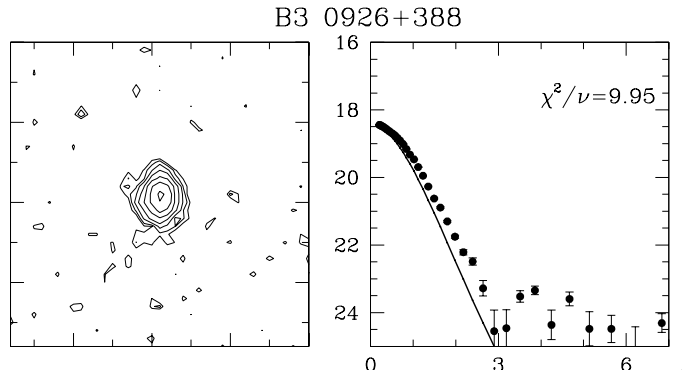

(b)
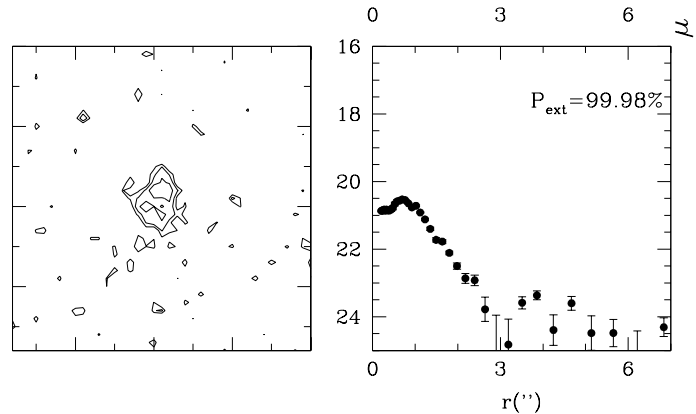

$r(")$

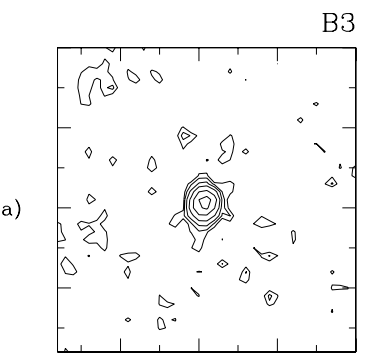

$32349+410$
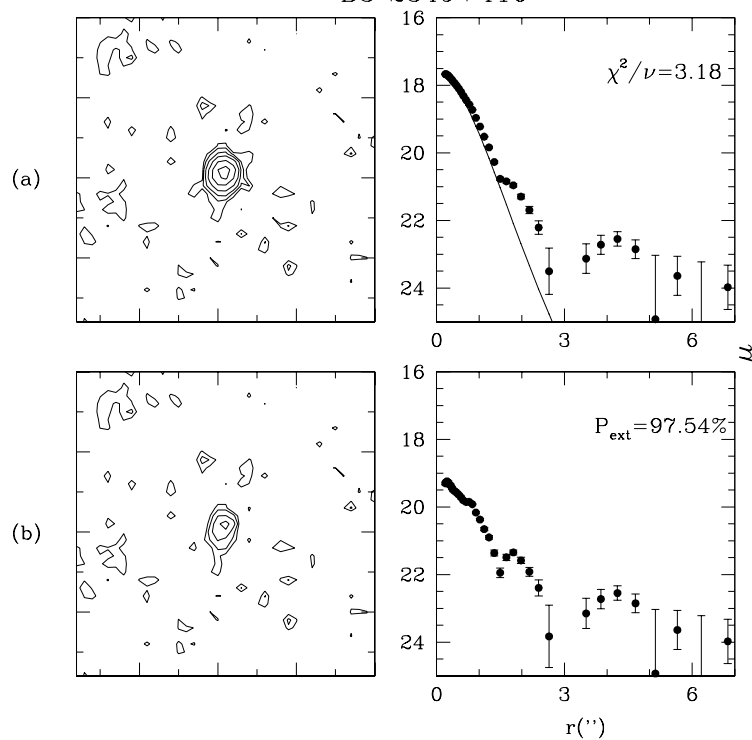

Fig. 3. continued.

This scenario describes the observed distribution accurately. Moreover, it explains the narrower distribution observed for the $3 \mathrm{C}$ radio galaxies due to the reduced range of radio luminosities (Lilly et al. 1985). In this context, we do not find radio galaxies as luminous in the NIR as the ultraluminous HGs observed here ( $\sim 13 \%$ of the sample) due to a selection effect. However, this scenario implies a relation between the HG luminosities and the radio power.

\subsection{Relation between the HG luminosity and the radio power}

Figure 5 shows the radio power of the 29 HGs of B3VLA quasars plus the $30 \mathrm{HGs}$ obtained from literature with published flux at $1460 \mathrm{MHz}$ (out of the original $40 \mathrm{HGs}$ ). The radio power has been obtained asuming a spectral index of $\alpha=-1$ for the radio emission, in all the objects. There is a clear correlation between these parameters for both the

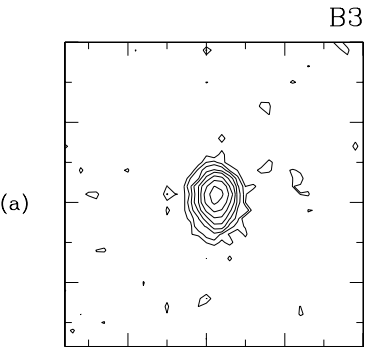

B3 $1315+396$
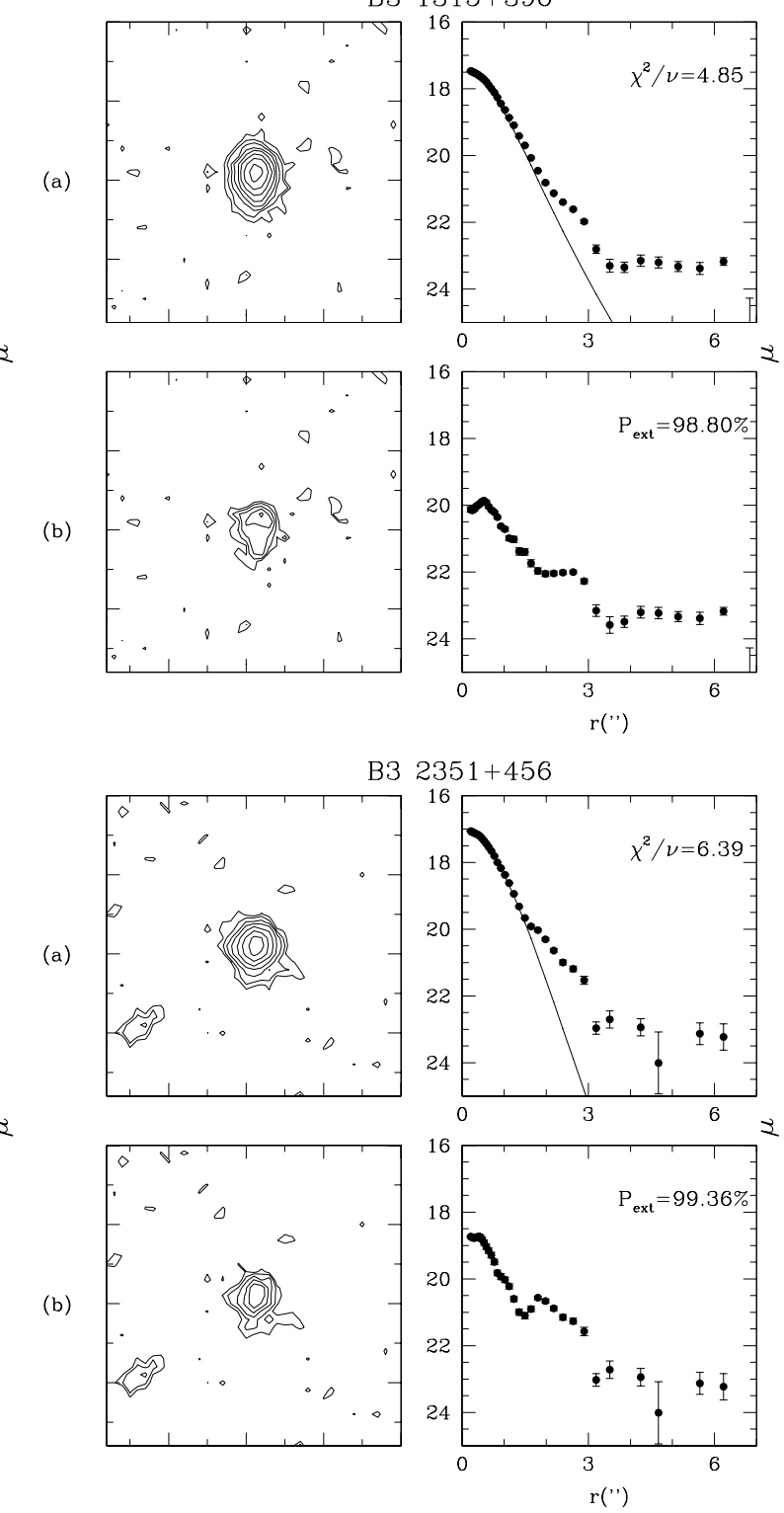

heterogeneous sample $(r=0.81 ; P>99.99 \%)$, and the B3VLA sample ( $r=0.70 ; P=99.88 \%)$. The B3-VLA subsample covers a range of radio-power/redshifts not very well covered before by data in the literature, extending the studies to more powerful/higher redshifts objects.

Figure 6 shows the distribution of radio power at $1460 \mathrm{MHz}$ against the absolute magnitude of the HGs. These parameters are clearly correlated, both for the heterogeneous sample $(r=-0.74 ; P>99.99 \%)$ and the B3-VLA subsample $(r=-0.56 ; P=99.81 \%)$. This correlation can be accounted for by the strong dependence on redshift shown by both parameters (the absolute magnitude of the host galaxies correlates with redshift, $r=-0.60 ; P>99.99 \%)$. Splitting the sample by the mean redshift, we find that there is still a correlation for the low- $z$ subsample $\left(r=-0.61 ; P>99.99 \% ; n_{\text {gal }}=37\right)$, although only a faint tendency for the high- $z$ subsample $(r=-0.32$, $\left.P=83.93 \% ; n_{\text {gal }}=22\right)$. It seems that there is an intrinsic relation between these two parameters, not induced by their 


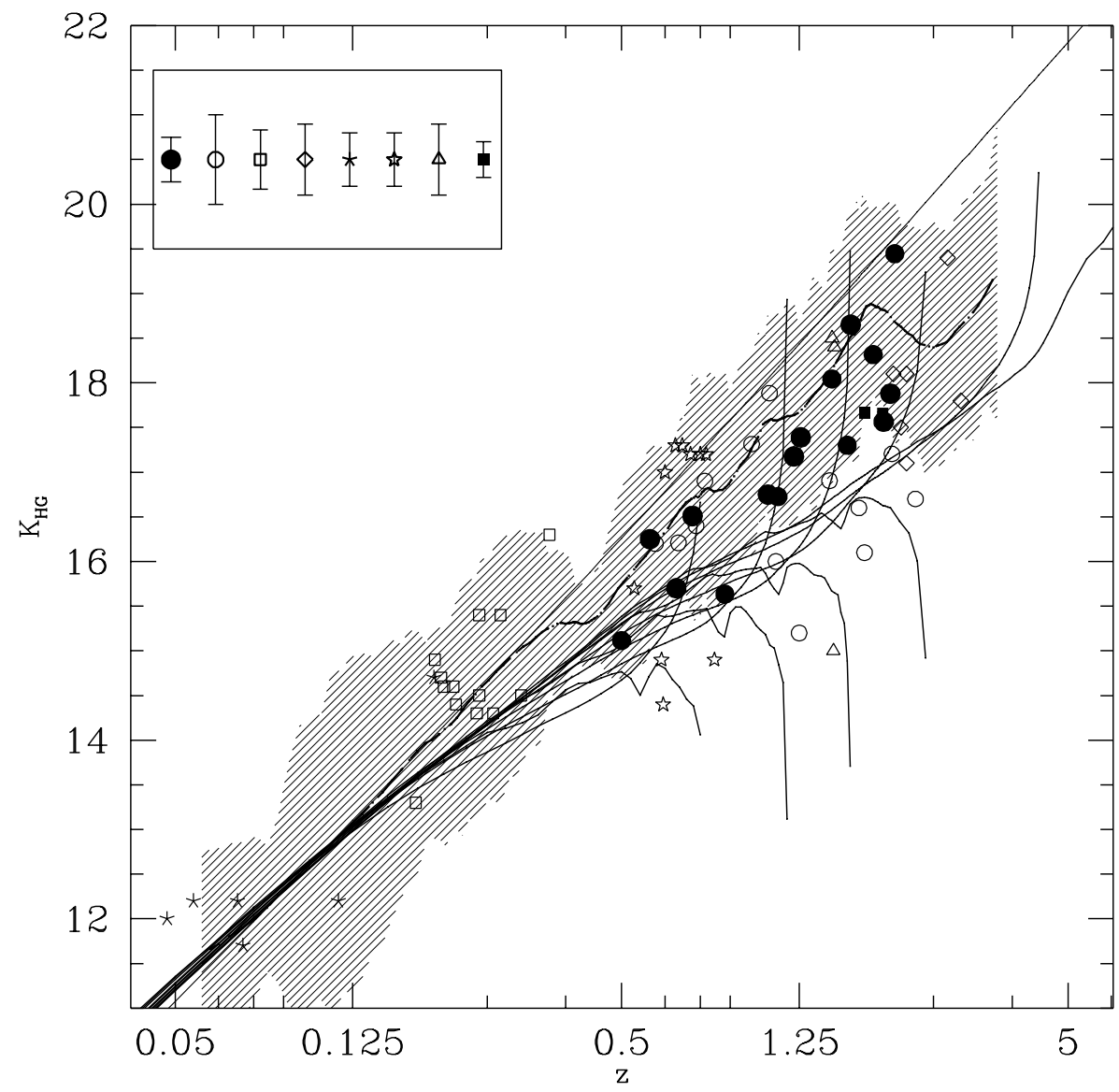

Fig. 4. $K$-band magnitude distribution with redshift for the HGs of radio quasars: circles for the B3-VLA (solid for new data and open for Paper I data), diamonds for Lehnert et al. (1992), open squares for Taylor et al. (1996), asterisks for Kotilainen et al. (1998), stars for Kotilainen \& Falomo (2000), triangles for Falomo et al. (2001) and solid squares for Kukula et al. (2001). The typical error of the magnitudes for the different samples is presented in the upper-left panel. The shaded region shows the parameter space occupied by radio galaxies of different samples (see text); the dashed line is the distribution of the mean values. The solid concave lines show the expected evolution of an elliptical galaxy with different formation redshifts $\left(z_{\text {for }}=7,4.5,2.5,1.5,1.0\right.$ and 0.7 , from right to left). The convex lines show the expected evolution of an galaxy with an ongoing burst with different formation redshifts ( $z_{\text {for }}=2.5,1.5,1.0$ and 0.7 , from right to left). The near straight solid line shows the expected magnitudes for a galaxy without evolution.

depencence with redshift. Similar tendencies have been described for radio galaxies (Ledlow \& Owen 1996; Rawlings et al. 1998; Lacy et al. 2000; Jarvis et al. 2001; Inskip et al. 2002; Willot et al. 2003), and radio-quiet quasars, of which the HGs at high $z(z \sim 2-3)$ are 2 mag fainter than powerful radio galaxies at the same redshift (Rawlings et al. 2001).

There is a simple explanation of why we may expect to find that stellar luminosities of HGs are correlated with radio luminosity. In essence, this comes down to the fact that more massive objects are generally more luminous. In this case there are several observed correlations that can be used to specify the relationship between stellar and radio luminosity. First, the stellar luminosity of ellipticals is known to correlate almost linearly with the central black hole mass (Magorrian et al. 1998), presumably because the stellar luminosity is very closely related to the stellar mass and there is a tight correlation between host and black hole masses (Gebhart et al. 2000; Ferrarese \& Merrit 2000). The radio luminosity is found to be linearly related to the narrow emission line luminosity (Willot et al. 2001) and therefore to the UV ionizing luminosity (Rawlings \& Saunders 1991; Willot et al. 1999; Carballo et al. 1999). The UV ionizing luminosity is due to the accretion of material onto the supermassive black hole. In fact, Franceschini et al. (1998) found a direct correlation between the radio luminosity and the mass of the central black hole, for a sample of low redshift galaxies. Therefore any correlation between stellar and radio luminosity is likely to have its cause in the fact that both parameters correlate positively with the black hole mass. McLure et al. (1999), and more recently Dunlop et al. (2003), reached a similar conclusion studying a sample of low- $z$ HGs. Our result extends theirs on the radio-loud sources to a wide range of redshifts $(0<z<3)$, increasing the statistical significance.

\subsection{Relation between the luminosities of the $H G$ and the central source}

There are several pieces of evidence supporting a scaling of the nuclear luminosity with HG luminosity. As we quoted above, Magorrian et al. (1998) found a correlation between the HG luminosity and the central black-hole mass. Since the accretion of matter is the main engine of the nuclear activity, it is expected that nuclear luminosity scales also with the black-hole mass. Indeed, Dunlop et al. (2003) have shown that radio-loud 


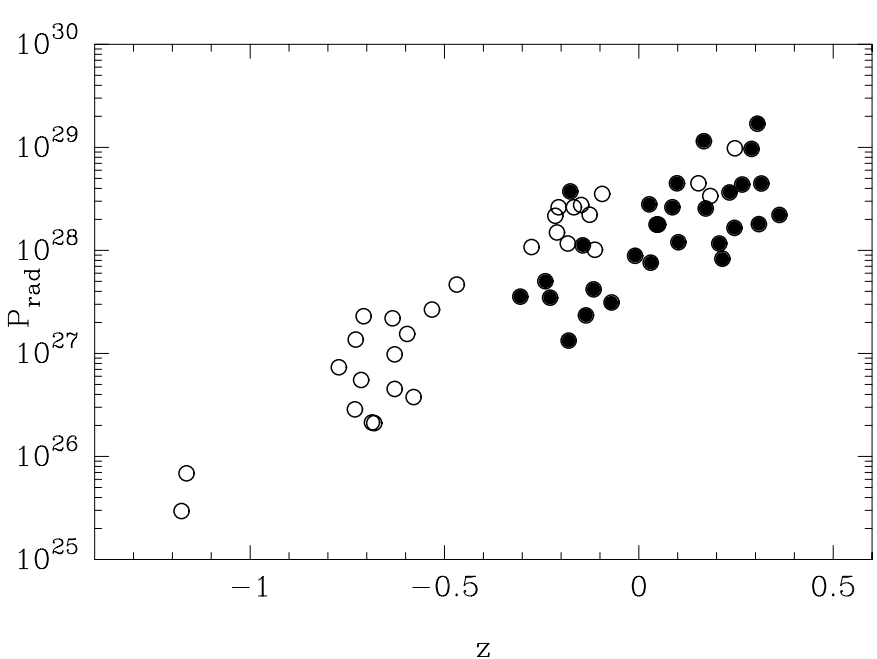

Fig. 5. Distribution of the radio power at $1460 \mathrm{MHz}$ against the redshift. The solid symbols are the HGs for the B3-VLA quasars; the open symbols are the HGs from the literature.

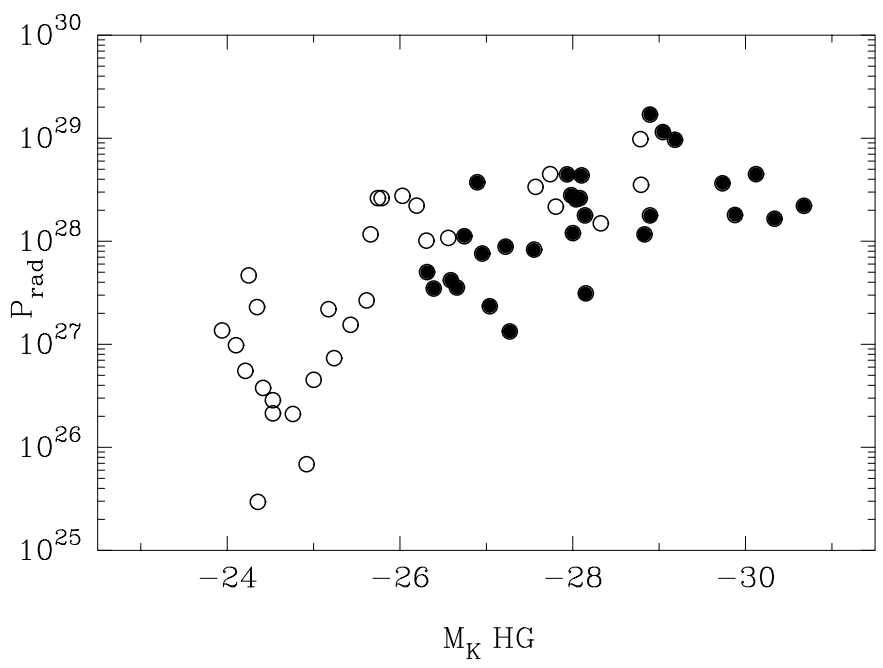

Fig. 6. Distribution of the radio power at $1460 \mathrm{MHz}$ against the absolute magnitude of the HGs. The solid symbols are the HGs for the B3-VLA quasars; the open symbols are the HGs from the literature.

quasars emit within a range of a 1-10\% of the Eddington luminosity. This luminosity is proportional to the black-hole mass. Therefore, a relation is expected between HG and nuclear component luminosities. On the other hand, hierarchical galaxy formation models of Kauffman \& Haehnelt (2000), in which they have addressed both the formation of bulges/elliptical galaxies and the formation and fueling of their associated black holes, predict a faint correlation between both luminosities.

Figure 7 shows the distribution of the absolute magnitudes of the HGs against the absolute magnitude of the nuclear source. There is a clear correlation for the heterogeneous sample $(r=0.56 ; P>99.99 \%)$ and the B3-VLA subsample $(r=0.57 ; P=99.88 \%)$. The strong depence that both parameters show with redshift could contribute significantly to this correlation (the absolute magnitude of the central sources also correlates with redshift, $r=-0.89 ; P>99.99 \%)$. We have determined the correlation coefficient for the low and high- $z$ subsamples, finding that there is still a correlation in both subsamples $(r=0.45 ; P=99.61 \%$ and $r=0.41 ; P=97.37 \%$, respectively). In order to remove the redshift effect we have determined the correlation coefficients for the apparent magnitudes, instead of the absolute magnitudes. Figure 8 shows the distribution of the apparent magnitudes of the HGs against the apparent magnitude of the nuclear source. We have found that the correlation is even stronger for the heterogeneous sample ( $r=0.74 ; P>99.99 \%)$, although only a slight correlation is found for the B3-VLA subsample, which covers a narrower range of apparent magnitudes $(r=0.34 ; P=96.79 \%)$. An intrinsic relation between both parameters rather than a redshift induced relation seems to be the correct explanation for the observed distributions.

The distributions in Figs. 7 and 8 could be induced by different biases. The QSO selection criteria as point-like sources could depopulate the lower-right region (bright HGs harboring a faint nuclear source). For the B3-VLA sample, the pointlike criterion was applied to POSS plate data (Vigotti et al. 1997). Similar data were used for the different samples included in our heterogeneous sample (e.g. Drinkwater et al. 1997 for the PKS). Taking into account the mean $B-K$ colours of the quasars and the elliptical galaxies $(\sim 2.5$ and $\sim 5$ mag respectively), we do not expect a significant effect of this bias on the observed distribution. E.g., a quasar with $B=17 \mathrm{mag}$, classified as point-like using the POSS plates could have a host galaxy that contributes $50 \%$ to its $K$-band flux, and what would be undetectable on POSS plates ( $\left.B_{\mathrm{HG}} \sim 20.5 \mathrm{mag}\right)$.

On the other hand, the upper-left region (faint HGs harboring a bright nuclear source) could be depopulated due to the incompleteness in the HG detection. Only HGs that contribute more than $10 \%$ to the total integrated flux are considered as real detections. This imposes an upper limit envelope on the observed distributions near to $K_{\mathrm{gal}}=K_{\text {nuc }}+2.5$. If this is the case we would expect an increase of the strength of the correlation with increasing incompleteness. However, as stated before, this strength is the same for the low- $z$ and high- $z$ subsamples, but the completeness decreases significantly ( $\sim 75 \%$ and $\sim 40 \%$ respectively, for the B3-VLA sample). Moreover, this argument could only be applied to the B3-VLA data. The heterogeneous sample was built using HG samples that are mainly complete or less affected by uncompleteness (due mainly to the lower redshift range they cover). Indeed, the distribution of B3-VLA data in Fig. 8 does not show a clear envelope at the predicted $2.5 \mathrm{mag}$ from the $K_{\text {Nucleus }}=K_{\mathrm{HG}}$ line.

These correlations confirm the tendency presented in previous articles for more powerful quasars to reside in more luminous hosts (e.g., Paper I, Kotilainen et al. 1998; Kotilainen \& Falomo 2000; Taylor et al. 1996). As we stated above, the most probable reason for this correlation is a relation of both luminosities with central black hole mass. This correlation is weaker or even absent in the more nearby, lower luminosity and radio-quiet AGNs (e.g. McLeod \& Rieke 1995; McLeod $\&$ Rieke 1994a,b; Percival et al. 2001). The smaller dynamical range of magnitudes covered by these samples could account for the absence of this correlation. 


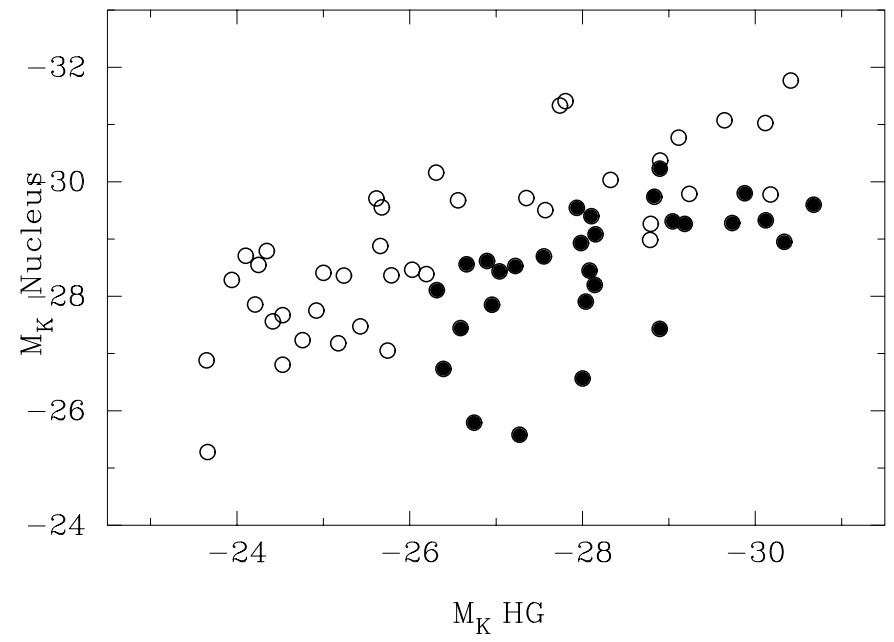

Fig. 7. Distribution of the $K$-band absolute magnitudes of the HGs against the absolute magnitude of the central source. The solid symbols are the HGs for the B3-VLA quasars; the open symbols are the HGs from the literature.

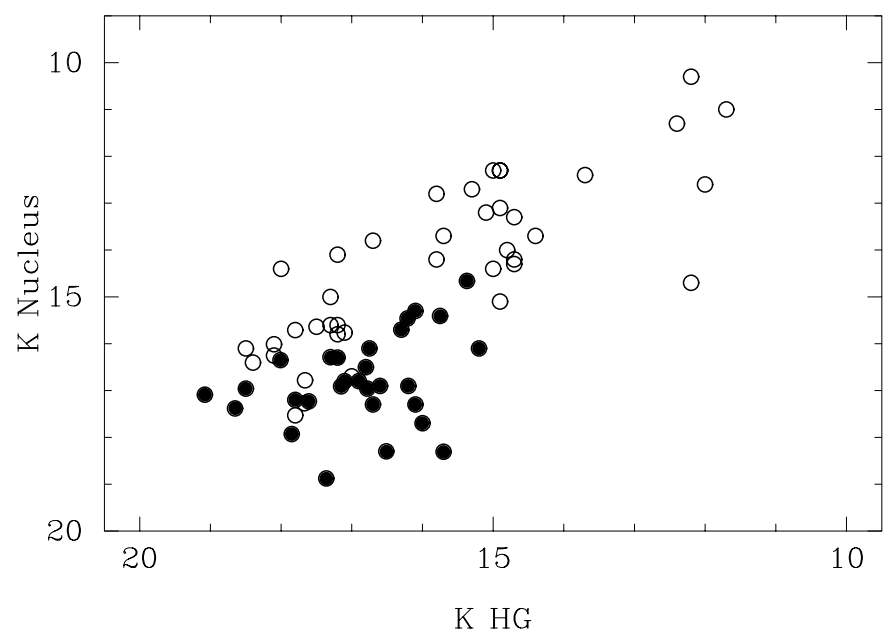

Fig. 8. Distribution of the $K$-band apparent magnitudes of the HGs against the apparent magnitude of the central source. The solid symbols are the HGs for the B3-VLA quasars; the open symbols are the HGs from the literature.

\subsection{Structural parameters of the HGs}

The mean absolute magnitude of the HGs is $\sim-27.02 \pm$ $1.81 \mathrm{mag}$, which corresponds to a luminosity of $\sim 3 L_{*}\left(M_{K}^{*}=\right.$ -25.86 from Mobasher et al. 1993). $\sim 75 \%$ of the objects are brighter than $L_{*}$. Their mean effective radius is $15.00 \pm$ $13.90 \mathrm{kpc}$ and all but one have $r_{\mathrm{e}}>3.5 \mathrm{kpc}$. Capaccioli et al. (1992) found that there is a limit to the size of the galaxies $(\sim 4.5 \mathrm{kpc})$, below which it is rare to find AGNs, BCG and/or cDs. As expected, $\sim 90 \%$ of the HGs of our sample are above this limit. They are large and luminous galaxies, similar to BCGs or cDs, which are in the range of the brightest known galaxies.

Elliptical galaxies show fundamental relations between their luminosities, their effective radii and their velocity dispersions (Hamabe \& Kormendy 1987; Mobasher et al. 1999). These relations define a plane in the three-dimensional space described above, the plane known as the Fundamental Plane
(FP). The projection of this plane on the luminosity-radius plane $\left(\mu_{\mathrm{e}}-r_{\mathrm{e}}\right)$ determines a relation between both parameters (Hoessel et al. 1987), with a slope near to $\sim 3$. This relation, of which the origin is still unknown, is shown by all elliptical galaxies, and limits the structural models presented for these objects. It has been suggested that episodes of collision/merging could produce this relation (Capaccioli et al. 1992).

We have determined the effective surface brightness of the HGs assuming a $r^{1 / 4}$ profile, correcting for cosmological dimming (e.g., Kolb \& Turner 1990). Figure 9 shows the distribution of the effective surface brightness with effective radius for the $50 \mathrm{HGs}$ of our sample with published effective radius. There is a clear correlation between both parameters, with a slope slightly larger than $3(\sim 4.6)$, roughly similar to the value reported by Taylor et al. (1996). Our sample is biased towards bright and large galaxies, especially at high redshift. The lack of small and faint galaxies could artificially increase the slope of the correlation. The dashed lines show the location on this plane of a galaxy with constant magnitude $(K=19 \mathrm{mag})$ and constant effective radius at different redshifts. Each line corresponds to a certain effective radius within the range $0.1^{\prime \prime}<r_{\mathrm{e}}<1.5^{\prime \prime}$. They represent the region of parameters where the incompleteness of the detection reaches $>80 \%$ for the B3-VLA data (solid circles). Similar exclusion regions could be defined for each sample plotted in the figure. We have restricted the linear regression analysis to the data less affected by this bias ( $\left.r_{\mathrm{e}}>10 \mathrm{kpc}, n=27 \mathrm{HGs}\right)$. We found a strong correlation $(r=0.80, P>99.99 \%)$, of the form:

$\mu_{\mathrm{e}}=15.64_{ \pm 0.60}+3.86_{ \pm 0.46} \log \left(r_{\mathrm{e}}\right)$

similar to the well-known relation for the elliptical galaxies.

\section{Relation between the absolute magnitude of the nuclear source and the radio power}

Figure 10 shows the distribution of the radio power of the quasars against the $K$-band absolute magnitude of the nuclear source. The parameters are clearly correlated for the heterogeneous sample $(r=0.57 ; P>99.99 \%)$ and the B3-VLA sample $(r=0.58 ; P=99.88 \%)$. This correlation could be induced by the dependence of both parameters on redshift. Splitting the sample in low $-z$ and high- $z$ subsamples by the mean redshift, we found that the correlation persists for both subsamples $(r=0.52, P=99.99 \%$ and $r=0.51, P=98.20 \%$ respectively). This suggests that it is not induced by the redshift. The derived relation between the $K$-band luminosity of the nuclear source and radio power is $L_{K, \mathrm{qso}} \propto P_{1460 \mathrm{MHz}}^{0.37 \pm 0.04}$. Carballo et al. (1999) found a similar relation between the optical-UV luminosity and radio power at $408 \mathrm{MHz}, L_{2400} \AA \propto P_{480 \mathrm{MHz}}^{0.52 \pm 0.10}$, for the B3-VLA quasars. These correlations imply a nearly one-to-one relation between the optical and NIR emission, which suggests that the principal mechanism of both kind of emissions is the same. The correlation between optical-NIR and radio emission in quasars has been discussed in Rawlings \& Saunders (1991), Serjeant et al. (1998), Willot et al. (1999), Carballo et al. (1999) and Simpson \& Rawlings (2000). It is outside the scope of the present study to discuss it in detail. 


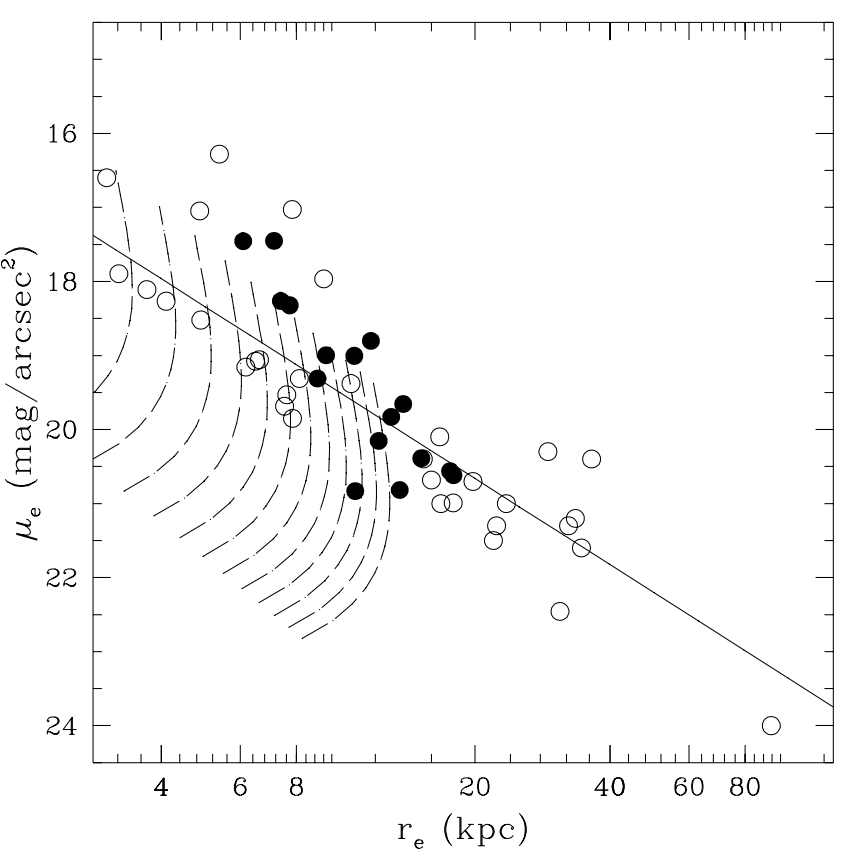

Fig. 9. Distribution of the effective surface brightness profile against the effective radius. The solid symbols are the HGs for the B3-VLA quasars; the open symbols are the HGs from the literature. The solid line show the best linear regression fit to the unbiased data. The dashed lines show the exclusion region for detection for the B3-VLA subsample.

\section{Discussion and conclusions}

We have presented in this study the $K$-band images of 31 radio quasars from the B3-VLA sample, with a redshift range between $z \sim 0.5$ and $z \sim 3.2$. A new procedure, based on the analysis of the surface brightness profile, has been proposed to estimate the flux contributions of both the point-like nucleus and the HG of the quasars. This procedure, tested on a wide sample of simulated images and field stars, makes it possible to assign a probability of being extended to each quasar, recover the flux and effective radius of the HG, classify it as elliptical or spiral, and restore the image of the HG. The simulations and tests on field stars set limits to the detection and reliability of the recovered parameters. A different procedure, used in Paper I (Carballo et al. 1998), has also been tested on the simulations, in order to allow the use of the combined data.

We have detected the HGs of 16 of the 31 quasars ( $50 \%$ of the sample), all of them with a probability of being extended of higher than $95 \%$, and 10 of more than $99 \%$. Together with data from Paper I, we have a sample of 29 HGs of B3-VLA quasars. Searching the literature, we have built a sample of heterogeneous HGs of radio-loud quasars that comprises 69 HGs. These HGs are large $\left(r_{\mathrm{e}} \sim 15 \mathrm{kpc}\right)$ and luminous ( $\sim 75 \%$ of them brighter than $L_{*}$ ) elliptical galaxies. They follow the $\mu_{\mathrm{e}}-r_{\mathrm{e}}$ relation for elliptical galaxies. About $\sim 45 \%$ of the B3-VLA HGs show distortions and tails in their images that could be evidence of a merging/colliding process. This result agrees with the hypothesis that considers a merging process as the origin or triggering mechanism of nuclear activity (e.g. Smith \& Heckman 1990; Hutching \& Neff 1992). There

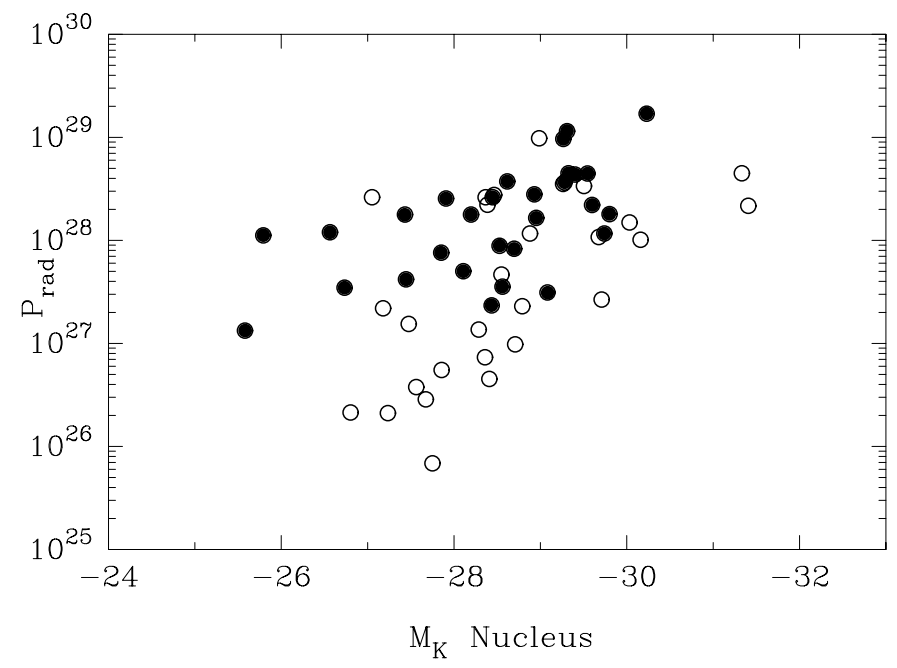

Fig. 10. Distribution of the radio power at $1460 \mathrm{MHz}$ against the absolute magnitude of the nuclear source. The solid symbols are the HGs for the B3-VLA quasars; the open symbols are the HGs from the literature.

is other evidence that supports this scenario: (i) the quasars are located in overdensities of galaxies compatible with being at the same redshifts (e.g. Sánchez \& González-Serrano 1999; Sánchez 2001), and in a spatial position where a collision is more likely to produce a merger (Sánchez \& González-Serrano 2002); (ii) the traces of recent star formation processes induced by interactions (e.g. Nolan et al. 2001; Canalizo \& Stockton 2000); (iii) the need of a violent process that produces an infall of mass in the inner region to explain nuclear activity (e.g. Bekki \& Noguchi 1994).

However, it is not clear that the fraction found could be considered as a significant excess of merger galaxies, even if all of them were confirmed. The fraction of merger/irregular galaxies in the local universe is $\sim 7 \%$, based on classical studies like the Shapley-Ames Catalog (Shapley \& Ames 1932). But this fraction is known to increase with redshift. E.g, van den Bergh et al. (1996) and Lee \& Hwang (2000) found that $39 \%$ of the galaxies on the HDF-N and HDF-S show irregular/merger morphologies. We do not have a proper sample of inactive galaxies with a similar redshift range and detailed morphological classification to known if the fraction of mergers found is or is not a significant excess.

All the HGs are elliptical galaxies morphologically similar to radio galaxies. The mean luminosity evolution along a wide range of redshifts $(0<z<3)$ is also similar to the evolution shown by radio galaxies. This evolution is well described by a model with a single burst of star formation at very high redshift $\left(z_{\text {for }}>7\right)$ and little or no evolution since that period. It seems that the galaxies have been formed beyond $z \sim 3$, without major burst process down to this redshift. This supports the unification schemes of radio sources, since both families of radio sources are similar in features that do not depend on the angle-of-view.

The HGs present a larger dispersion in mean evolution compared with the dispersion found in radio galaxies, especially beyond $z>1$. This dispersion is most probably due to a 
correlation between the absolute magnitude of the HGs and the radio power. Therefore, the most powerful radio quasars inhabit the most luminous HGs. The absolute magnitudes of the host and nuclear source (and this last parameter and radio power) are also correlated. These correlations are most probably due to a correlation of all three parameters with the central black hole mass (McLure et al. 1999). Dunlop et al. (2003) found that this tendency is seen both in radio-loud and radio-quiet samples, which could imply that the same mechanism produces the radio emission in both kinds of AGN.

In a toy scenario, succesive merging processes could increase the mass of the spheroidal component of the HG, increasing its luminosity. These merging processes would feed the nuclear AGN, increasing also the mass of the central black hole. This will also increase the nuclear luminosity and radio power. This scenario is consistent with the result that indicates that radio-quiet quasars inhabit less-populated areas than radioloud quasars (Hall et al. 1998). Sánchez \& González-Serrano (1999) and Sánchez \& González-Serrano (2002) have found that QSOs inhabit the outer regions of clusters (instead of the core) where a collision is more likely to produce a merger. Recent morphological analysis of HGs of different families of AGNs at $z<0.3$ found no traces of recent merging processes (Dunlop et al. 2003). This could indicate an evolution in the merging rate, although much more detailed studies are needed before a conclusion can be reached.

The global scenario suggested by the results presented in this article has to be tested. The first obvious step would be to increase the fraction of detected HGs in order to reduce the biases, and to extend the studies to other samples with higher and lower radio powers. Photometric studies of the HGs at other wavelengths, and spectroscopy of them and their near companions are needed to determine better the evolution of these galaxies, and search for possible traces of merging processes.

Acknowledgements. We thank the anonymous referee for his valuable and interesting comments that has increased the quality of this article.

We thank L. Cayón and I. Ferreras for making available the GISSEL code, and for their support with its operation. We thank all Calar Alto observatory staff for the friendly support and the inestimable help in the successive observing runs. S. F. Sánchez thanks Danny Lennon (ING Head of the astronomy group) for their support in the realization of this research. S. F. Sánchez thanks Mariano Tejedor (Eresmas SA) for his kind help in letting him have time and computers to work on this project. This project has been partially funded by grants from the Spanish Ministerio de Educacion y Cultura and Ministerio de Ciencia y Tecnologia, with the projects PB98-0409 and AYA2002-03326, respectively. This project has be partially founded by the Euro3D Training Network on Integral Field Spectroscopy, funded by the European Commission under contract No. HPRN-CT2002-00305.

3.5 m CAHA Visiting Astronomer, German-Spanish Astronomical Centre, Calar Alto, operated by the Max-Planck-Institute for Astronomy, Heidelberg, jointly with the Spanish National Commission for Astronomy.

Based on observations made with the WHT operated on the island of La Palma by the Isaac Newton Group in the Spanish Observatorio del Roque de los Muchachos of the Instituto de Astrofisica de Canarias. The authors want to thank M. Bolzonella for his help with the GISSEL code and the isochrone spectral energy distribution data.
The authors want to thank C.R.Benn for his inestimable help. His comments, tests and objective criticism concerning the data have strongly influenced the final version of the article.

\section{References}

Antonucci, R. 1993, ARA\&A, 31, 473

Aretxaga, I., LeMignant, D., Melnick, J., Terlevich, R. J., \& Boyle, B. J. 1998, MNRAS, 298, 13

Bahcall, J. N., Kirhakos, S., \& Schneider, D. P. 1994, ApJ, 435, L11

Bahcall, J. N., Kirhakos, S., \& Schneider, D. P. 1995a, ApJ, 447, L1

Bahcall, J. N., Kirhakos, S., \& Schneider, D. P. 1995b, ApJ, 450, 486

Bahcall, J. N., Kirhakos, S., \& Schneider, D. P. 1996, ApJ, 457, 557

Bahcall, J. N., Kirhakos, S., Saxe, D. H., \& Schneider, D. P. 1997, ApJ, 479

Bekki, K., \& Noguchi, M. 1994, A\&A, 290, 7

Bolzonella, M., Miralles, J. M., \& Pelló, R. 2000, A\&A, 363, 476

Boroson, T. A., \& Oke, J. B. 1982, Nature, 296, 397

Boroson, T. A., \& Oke, J. B. 1984, ApJ, 281, 535

Bruzual, A. G., \& Charlot, S. 1993, ApJ, 405, 558

Canalizo, G., \& Stockton, A. 2000, ApJ, 328, 201

Canalizo, G., \& Stockton, A. 2001, ApJ, 555, 719

Capaccioli, M., Caon, N., \& D'Onofrio, M. 1992, MNRAS, 259, 323

Carballo, R., Sánchez, S. F., González-Serrano, J. I., Benn, C. R., \& Vigotti, M. 1998, AJ, 115, 1234

Carballo, R., González-Serrano, J. I., Benn, C. R., Sánchez, S. F., \& Vigotti, M. 1999, MNRAS, 306, 137

Casali, M. M., \& Hawarden, T. G. 1992, UKIRT Newslett., 4, 33

Courbin, F., Letawe, G., Magain, P., et al. 2002, A\&A, 394, 863

de Vaucouleurs, G. 1948, Ann. Astrophys., 11, 247

de Vries, W. H., O'Dea, C. P., Barthel, P. D., et al. 2000, AJ, 120, 2300

Disney, M., Boyce, P. J., Blades, J. C., et al. 1995, Nature, 376, 150

Drinkwater, M. J., Webster, R. L., Francis, P. J., et al. 1997, MNRAS, 284, 85

Dunlop, J. S., \& Peacock, J. A. 1993, MNRAS, 263, 936

Dunlop, J. S., Taylor, G. L., Hughes, D. H., \& Robson, E. L. 1993, MNRAS, 264, 445

Dunlop, J. S., McLure, R. J., Kukula, M. J., et al. 2003, MNRAS, 340, 1095

Eales, S. A., \& Rawlings, S. 1996, ApJ, 460, 68

Ellingson, E., Yee, H. K. C., \& Green, R. F. 1991, ApJ, 371, 49

Falomo, R., Kotilainen, J., \& Treves, A. 2001, ApJ, 547, 124

Ferrarese, L., \& Merritt, D. 2000, ApJ, 539, L9

Franceschini, A., Vercellone, S., \& Fabian, A. C. 1998, MNRAS, 397, 817

Freeman, K. C. 1970, ApJ, 160, 812

Gebhardt, K., Bender, R., Bower, G., et al. 2000, ApJ, 539, L13

González-Serrano, J. I., Carballo, R., \& Pérez-Fournon, I. 1993, AJ, 105,1710

Hall, P. B., Green, R. F., \& Cohen, M. 1998, ApJS, 119, 1

Hamabe, M., \& Kormendy, J., Structure and Dynamics of Elliptical Galaxies, ed. T. de Zeeuw (Dordrecht: Reidel), IAU Symp., 127, 379

Heckman, T. M., Miley, G. K., Lehnert, M. D., \& van Breugel, W. 1991, ApJ, 370, 78

Hoessel, J. G., Oegerle, W. R., \& Schneider, D. P. 1987, AJ, 94, 1111

Hughes, D. H., Kukula, M. J., Dunlop, J. S., \& Boroson, T. 2000, MNRAS, 316, 204

Hutchings, J. B., \& Neff, S. G. 1992, AJ, 104,1

Hutchings, J. B., \& Neff, S. G. 1997, AJ, 113, 550

Hutchings, J. B., \& Morris, S. C. 1995, AJ, 109, 1541

Hutchings, J. B., Holtzman, J., Sparks, W. B., et al. 1994, ApJ, 429, L1 
Hutchings, J. B. 1995, Nature, News \& Views, 376,118

Hutchings, J. B., Frenette, D., Hanisch, R., et al. 2002, AJ, 123, 2936

Inskip, K. J., Best, P. N., Longair, M. S., \& MacKay, D. J. C. 2002, MNRAS, 329, 277

Jarvis, M. J., Rawlings, S., Eales, S. A., et al. 2001, MNRAS, 326, 1585

Jedrzejewski, R. I. 1987, MNRAS, 226, 747

Kauffmann, G., \& Haehnelt, M. 2000, MNRAS, 311, 576

Kirhakos, S., Bahcall, J. N., Schneider, D. P., \& Kristian, J. 2001, ApJ, 520,67

Kolb, E. W., \& Turner, M. S. 1990, The Early Universe (Addison-Wesley Publishing Company), 42

Kormendy, J., \& Richstone, D. 1995, ARA\&A, 33, 581

Kotilainen, J. K., Falomo, R., \& Scarpa, R. 1998, A\&A, 332, 503

Kotilainen, J. K., \& Falomo, R. 2000, A\&A, 364, 70

Kukula, M. J., Dunlop, J. S., Hughes, D. H., Taylor, G., \& Boroson, T. 1997, Quasar Hosts, ed. D. L. Clements, \& I. Pérez-Fournón, ESO-IAC Conf., 177

Kukula, M. J., Dunlop, J. S., McLure, R. J., Miller, L., et al. 2001, MNRAS, 325, 1533

Lacy, M., Bunker, A. J., \& Ridgway, S. E. 2000, AJ, 120, 68

Ledlow, M. J., \& Owen, F. N. 1996, AJ, 112, 9

Lee, M. G., \& Hwang, N. 2000, ISASS, 14, 131

Lehnert, M. D., Heckman, T. M., Chambers, K. C., \& Miley, G. K. 1992, ApJ, 393, 68

Lehnert, M., Miley, G. K., Sparks, W. B., et al. 1999, ApJS, 123, 351

Lehnert, M., Matthew, D., van Breugel, W. J. M., Heckman, T. M., \& Miley, G. K. 1999, ApJS, 124, 11

Lilly, S. J. 1989, ApJ, 340, 77

Lilly, S. J., Longair, M. S., \& Allington-Smith, J. R. 1985, MNRAS, 215, 37

MacKenty, J. W. 1990, ApJS, 72, 231

Magorrian, J., Tremaine, S., Richstone, D., et al. 1998, AJ, 115, 2285

McLeod, K. K., \& Rieke, G. H. 1994, ApJ, 420, 58

McLeod, K. K., \& Rieke, G. H. 1994, ApJ, 431, 137

McLeod, K. K., \& Rieke, G. H. 1995, ApJ, 454, L77

McLeod, K. K., \& McLeod, B. A. 2001, ApJ, 546, 782

McLure, R. J., Kukula, M. J., Dunlop, J. S., et al. 1999, MNRAS, 308, 377

McLure, R. J., \& Dunlop, J. S. 2002, MNRAS, 331, 795

Meurer, G. R., Heckman, T. M., Leitherer, C., et al. 1995, ApJ, 110, 2665

Moffat, A. F. J. 1969, A\&A, 3, 455

Mobasher, B., Sharples, R. M., \& Ellis, R. S. 1993, MNRAS, 263, 560
Mobasher, B., Guzmán, R., Aragón-Salamanca, A., \& Zepf, S. 1999, MNRAS, 304, 225

Nolan, L. A., Dunlop, J. S., Kukula, M. J., et al. 2001, MNRAS, 323, 308

Percival, W. J., Miller, L., McLure, R. J., \& Dunlop, J. S. 2001, MNRAS, 322, 843

Rawlings, S., \& Saunders, R. 1991, Nature, 349, 138

Rawlings, S., Blundell, K. M., Lacy, M., Willot, C. J., \& Eales, S. A. 1998, Proc. of the workshop, Observational Cosmology with Radio Surveys (Kluwer), ASSL, 226, 171 [astro-ph/9704151]

Ridgway, S. E., \& Stockton, A. 1997, AJ, 114, 511

Ridgway, S. E., Heckman, T. M., Calzetti, D., \& Lehnert, M. 2001, ApJ, 550, 122

Riegler, M. A., Lilly, S. J., Stockton, A., Hammer, F., \& Le Fèvre, O. 1992, ApJ, 385, 61

Salpeter, E. E. 1955, ApJ, 121, 161

Sánchez, S. F. 2001, Ph.D. Thesis, Univ. of Cantabria

Sánchez, S. F., \& Gonzalez-Serrano, J. I. 1999, A\&A, 352, 395

Sánchez, S. F., \& Gonzalez-Serrano, J. I. 2002, A\&A, 396, 773

Serjeant, S., Rawlings, S., Maddox, S. J., et al. 1998, MNRAS, 294, 494

Shapley, H., \& Ames, A. 1932, Harvard Ann., 88, 2

Simpson, C., \& Rawlings, S. 2000, MNRAS, 317, 1023

Smith, E. P., \& Heckman, T. M. 1990, ApJ, 348, 38

Smith, E. P., Heckman, T. M., Bothun, G. D., Romanishin, W., \& Balick, B. 1986, ApJ, 306, 64

Stockton, A., \& Ridgway, S. E. 2001, 554, 1012

Taylor, G. T., Dunlop, J. S., Hughes, D. H., \& Robson, E. I. 1996, MNRAS, 283, 930

Urry, C. M., \& Padovani, P. 1995, PASP, 107, 803

van den Bergh, S., Abraham, R. G., Ellis, R. S., Tanvir, N. R., \& Glazebrook, K. G. 1996, AJ, 112, 359

Van Breugel, W., de Breuck, C., Stanford, S. A., et al. 1999, ApJ, 518,61

Véron-Cetty, M. P., \& Woltjer, L. 1990, AA, 236, 69

Vigotti, M., Vettolani, G. V., Merighi, R., Lahulla, J. F., \& Pedani, M. 1997, A\&AS, 123, 1

Whitmore, B. C, Zhang, Q., Leitherer, C., et al. 1999, ApJ, 118, 1551

Willot, C. J., Rawlings, S., Blundell, K. M., \& Lacy, M. 1999, MNRAS, 309, 1017

Willot, C. J., Rawlings, S., \& Blundell, K. M. 2001, MNRAS, 324, 1

Willot, C. J., Rawlings, S., Jarvis, M. J., \& Blundell, K. M. 2003, MNRAS, 339, 173 